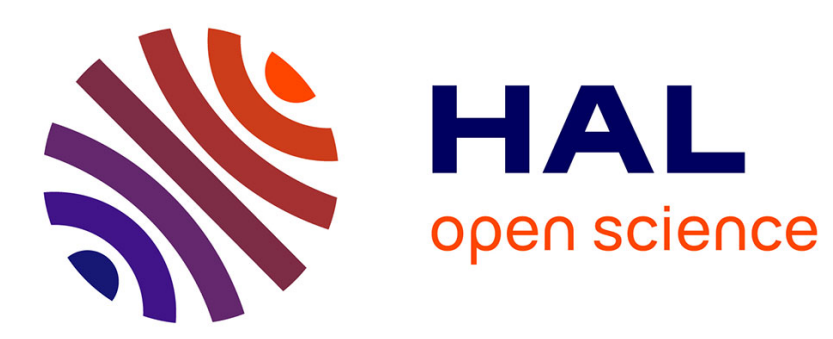

\title{
Homogeneous Lyapunov functions: from converse design to numerical implementation
}

Denis Efimov, Rosane Ushirobira, Jaime A Moreno, Wilfrid Perruquetti

\section{To cite this version:}

Denis Efimov, Rosane Ushirobira, Jaime A Moreno, Wilfrid Perruquetti. Homogeneous Lyapunov functions: from converse design to numerical implementation. SIAM Journal on Control and Optimization, 2018, 56 (5), pp.24. 10.1137/17M113753X . hal-01851761

\section{HAL Id: hal-01851761 \\ https://hal.inria.fr/hal-01851761}

Submitted on $30 \mathrm{Jul} 2018$

HAL is a multi-disciplinary open access archive for the deposit and dissemination of scientific research documents, whether they are published or not. The documents may come from teaching and research institutions in France or abroad, or from public or private research centers.
L'archive ouverte pluridisciplinaire HAL, est destinée au dépôt et à la diffusion de documents scientifiques de niveau recherche, publiés ou non, émanant des établissements d'enseignement et de recherche français ou étrangers, des laboratoires publics ou privés. 


\title{
Homogeneous Lyapunov functions: from converse design to numerical implementation
}

\author{
D. Efimov, R. Ushirobira, J. A. Moreno, W. Perruquetti
}

\begin{abstract}
The problem of the synthesis of a homogeneous Lyapunov function for an asymptotically stable homogeneous system is studied. First, for systems with nonnegative degree of homogeneity, several expressions of homogeneous Lyapunov functions are derived, which depend explicitly on the supremum or the integral (over finite or infinite intervals of time) of the system solutions. Second, a numeric procedure is proposed, which ensures the construction of a homogeneous Lyapunov function. The analytical results are illustrated by simulations.
\end{abstract}

\section{INTRODUCTION}

Stability analysis is one of the central problems in the domains of control and dynamical systems [1], [4], [8], [13], [15], [17], [19], [22], [30]. The main approach to check the stability of a nonlinear dynamical system consists in applying the Lyapunov function method, that gives necessary and sufficient conditions for stability. The main issue with the application of this method is that there exists no procedure to design such a Lyapunov function for a generic dynamical system. There exist analytical methods to construct a Lyapunov function for linear or Lurie systems [18], and several numerical approaches have been proposed for linear and partially linear (linear parameter-varying) systems [5], see also the survey [14]. The sum of squares approach also can be effectively applied for some classes of systems (like polynomial ones) [25].

Homogeneous systems form a subclass of nonlinear systems [1], [2], that include linear systems as a special case. These systems inherit many useful properties in a generic setting from the linear case: scalability of solutions, estimation of the rate of convergence and robustness [3], [2]. A remarkable fact about homogeneous systems is that they possess homogeneous Lyapunov functions [33], [29]. However, no numeric routine has been proposed so far to construct such a Lyapunov function using homogeneity properties (the works [33], [29] assumed that a Lyapunov function is already given, and a special transformation is then presented providing homogeneity property), and the present paper is going to fill this gap.

The aim of this work is to propose several homogeneous Lyapunov functions based on converse Lyapunov function methods, and also to develop numerical tools for calculating an explicit homogeneous Lyapunov function for a given nonlinear homogeneous system. The proposed results are based on an important feature of homogeneous Lyapunov functions: their construction and analysis can be performed on a sphere only, making calculations simpler. The numerical algorithms obtained here for constructing homogeneous Lyapunov functions can be used for checking the stability of homogeneous systems (since a system on a sphere can be approximated by a homogeneous one [10], this approach can be applied to a wide class of nonlinear systems locally). Their extension can also be implemented for control design using universal formulas [31], which are implicitly dependent on (control) Lyapunov functions.

The outline of this paper is as follows. The notation and preliminary results are introduced in sections II and III. The analysis of robustness of homogeneous Lyapunov functions is carried out in Section IV. Several explicit analytic expressions for homogeneous Lyapunov functions are proposed in Section V. Numerical routines using those expressions to design a homogeneous Lyapunov function are presented in Section VI. The proposed approach for constructing homogeneous Lyapunov functions is illustrated by numerical examples in Section VII. Concluding remarks and discussion appear in Section VIII.

\section{NOTATION}

- $\mathbb{R}_{+}=\{x \in \mathbb{R}: x \geq 0\}$, where $\mathbb{R}$ is the set of real numbers, $\mathbb{N}$ is the set of natural numbers.

- $|\cdot|$ denotes the absolute value in $\mathbb{R},\|\cdot\|$ denotes the Euclidean norm on $\mathbb{R}^{n}$ (for $n \in \mathbb{N}$ ), $\|x\|_{\mathcal{A}}=\inf _{\xi \in \mathcal{A}}\|x-\xi\|$ is the distance from a point $x \in \mathbb{R}^{n}$ to a set $\mathcal{A} \subset \mathbb{R}^{n}$.

- A continuous function $\alpha: \mathbb{R}_{+} \rightarrow \mathbb{R}_{+}$belongs to the class $\mathcal{K}$ if $\alpha(0)=0$ and the function is strictly increasing. The function $\alpha: \mathbb{R}_{+} \rightarrow \mathbb{R}_{+}$belongs to the class $\mathcal{K}_{\infty}$ if $\alpha \in \mathcal{K}$ and it increases to infinity. A continuous function

The preliminary version of this work has been partially presented in [12] without proofs and with a different numeric procedure.

D. Efimov, R. Ushirobira and W. Perruquetti are with Inria, Univ. Lille, CNRS, UMR 9189 - CRIStAL, Centrale Lille, F-59000 Lille, France. J. A. Moreno is with Instituto de Ingeniería, UNAM, Coyoacán, 04510 México DF, Mexico.

This work was partially supported by HoTSMoCE Inria Associate Team and by ANR 15 CE23 0007 (Project Finite4SoS). 
$\beta: \mathbb{R}_{+} \times \mathbb{R}_{+} \rightarrow \mathbb{R}_{+}$belongs to the class $\mathcal{K} \mathcal{L}$ if $\beta(\cdot, t) \in \mathcal{K}_{\infty}$ for each fixed $t \in \mathbb{R}_{+}$and $\lim _{t \rightarrow+\infty} \beta(s, t)=0$ for each fixed $s \in \mathbb{R}_{+}$.

- A sequence of integers $1,2, \ldots, n$ is denoted by $\overline{1, n}$.

\section{PReliminaries}

Consider the following nonlinear system:

$$
\dot{x}(t)=f(x(t)), t \geq 0,
$$

where $x(t) \in \mathbb{R}^{n}$ is the state and $f: \mathbb{R}^{n} \rightarrow \mathbb{R}^{n}$ ensures forward existence and uniqueness of the system solutions at least locally in time, $f(0)=0$. For an initial condition $x_{0} \in \mathbb{R}^{n}$ denote the corresponding solution by $X\left(t, x_{0}\right)$ for any $t \geq 0$ for which the solution exists (the solutions are understood in the Carathéodory sense). A set $\mathcal{A} \subset \mathbb{R}^{n}$ is called forward invariant for (1) if $x_{0} \in \mathcal{A}$ implies that $X\left(t, x_{0}\right) \in \mathcal{A}$ for all $t \geq 0$.

\section{A. Stability definitions}

Following [1], [18], [21], [27], let $\Omega$ be an open neighborhood of a forward invariant set $\mathcal{A} \subset \mathbb{R}^{n}$ of (1).

Definition 1. For the system (1), the set $\mathcal{A}$ is said to be:

(a) Lyapunov stable if for any $x_{0} \in \Omega$ the solution $X\left(t, x_{0}\right)$ is defined for all $t \geq 0$, and for any $\epsilon>0$ there is $\delta>0$ such that for any $x_{0} \in \Omega$, if $\left\|x_{0}\right\|_{\mathcal{A}} \leq \delta$ then $\left\|X\left(t, x_{0}\right)\right\|_{\mathcal{A}} \leq \epsilon$ for all $t \geq 0$;

(b) asymptotically stable if it is Lyapunov stable and for any $\kappa>0$ and $\epsilon>0$ there exists $T(\kappa, \epsilon) \geq 0$ such that for any $x_{0} \in \Omega$, if $\left\|x_{0}\right\|_{\mathcal{A}} \leq \kappa$ then $\left\|X\left(t, x_{0}\right)\right\|_{\mathcal{A}} \leq \epsilon$ for all $t \geq T(\kappa, \epsilon)$;

(c) finite-time stable if it is Lyapunov stable and finite-time converging from $\Omega$, i.e. for any $x_{0} \in \Omega$ there exists $0 \leq T<+\infty$ such that $X\left(t, x_{0}\right) \in \mathcal{A}$ for all $t \geq T$. The function $\mathcal{T}_{\mathcal{A}}\left(x_{0}\right)=\inf \left\{T \geq 0: X\left(t, x_{0}\right) \in \mathcal{A} \forall t \geq T\right\}$ is called the settling time of the system (1);

(d) fixed-time stable if it is finite-time stable and $\sup _{x_{0} \in \Omega} \mathcal{T}_{\mathcal{A}}\left(x_{0}\right)<+\infty$.

The set $\Omega$ is called the domain of stability/attraction.

If $\Omega=\mathbb{R}^{n}$, then the corresponding properties are called global Lyapunov/asymptotic/finite-time/fixed-time stability of (1) at $\mathcal{A}$.

For a $V: \mathbb{R} \rightarrow \mathbb{R}$ denote the upper Dini derivative:

$$
\dot{V}^{+}(t)=\limsup _{h \rightarrow 0^{+}} \frac{V(t+h)-V(t)}{h}, \forall t \in \mathbb{R}_{+} .
$$

If $V$ is locally Lipschitz continuous then $\dot{V}^{+}$is finite, and if $V$ is differentiable then $\dot{V}^{+}$is the usual derivative of $V$. For $V: \mathbb{R}^{n} \rightarrow \mathbb{R}$ the generalized directional derivative at $x \in \mathbb{R}^{n}$ in the direction $d \in \mathbb{R}^{n}$ is defined by:

$$
D^{+} V(x) d=\limsup _{\substack{y \rightarrow x \\ h \rightarrow 0^{+}}} \frac{V(y+h d)-V(y)}{h} .
$$

\section{B. Weighted homogeneity}

Following [1], [33], for strictly positive real numbers $r_{i}(i=\overline{1, n})$ called weights and $\lambda>0$, one can define:

- the vector of weights $\mathbf{r}=\left(r_{1}, \ldots, r_{n}\right)^{T}, r_{\max }=\max _{1 \leq j \leq n} r_{j}$ and $r_{\min }=\min _{1 \leq j \leq n} r_{j}$;

- the dilation matrix function $\Lambda_{r}(\lambda)=\operatorname{diag}\left(\lambda^{r_{i}}\right)_{i=1}^{n}$ (note that $\forall x \in \mathbb{R}^{n}$ and $\forall \lambda>0$ we have $\Lambda_{r}(\lambda) x=$ $\left.\left(\lambda^{r_{1}} x_{1}, \ldots, \lambda^{r_{i}} x_{i}, \ldots, \lambda^{r_{n}} x_{n}\right)^{T}\right)$;

- the $\mathbf{r}$-homogeneous norm of $x \in \mathbb{R}^{n}$ is $\|x\|_{r}=\left(\sum_{i=1}^{n}\left|x_{i}\right|^{\frac{\rho}{r_{i}}}\right)^{\frac{1}{\rho}}$ for $\rho \geq r_{\max }$ (it is not a norm in common sense since the triangle inequality does not hold);

- for $s>0$ the sphere and the ball in the homogeneous norm $S_{r}(s)=\left\{x \in \mathbb{R}^{n}:\|x\|_{r}=s\right\}$ and $B_{r}(s)=\left\{x \in \mathbb{R}^{n}\right.$ : $\left.\|x\|_{r} \leq s\right\}$, respectively.

Definition 2. A function $g: \mathbb{R}^{n} \rightarrow \mathbb{R}$ is $\mathbf{r}$-homogeneous of degree $\mu \in \mathbb{R}$ if $\forall x \in \mathbb{R}^{n}$ and $\forall \lambda>0$ we have:

$$
\lambda^{-\mu} g\left(\Lambda_{r}(\lambda) x\right)=g(x) .
$$

A vector field $f: \mathbb{R}^{n} \rightarrow \mathbb{R}^{n}$ is $\mathbf{r}$-homogeneous of degree $\nu \in \mathbb{R}$, with $\nu \geq-r_{\min }$ if $\forall x \in \mathbb{R}^{n}$ and $\forall \lambda>0$ we have:

$$
\lambda^{-\nu} \Lambda_{r}^{-1}(\lambda) f\left(\Lambda_{r}(\lambda) x\right)=f(x),
$$

which is equivalent to the $i$-th component of $f$ being a $\mathbf{r}$-homogeneous function of degree $r_{i}+\nu$. 
The system (1) is $\mathbf{r}$-homogeneous of degree $\nu$ if the vector field $f$ is $\mathbf{r}$-homogeneous of degree $\nu$.

For $\mathbf{r}$-homogeneous system (1) of degree $\nu$, the solutions also admit a kind of homogeneity as functions of time [1], i.e. $\forall x_{0} \in \mathbb{R}^{n}$ and $\forall t \in \mathbb{R}$ :

$$
X\left(t, \Lambda_{r}(\lambda) x_{0}\right)=\Lambda_{r}(\lambda) X\left(\lambda^{\nu} t, x_{0}\right) \quad \forall \lambda>0 .
$$

By its definition, $\|\cdot\|_{r}$ is an $\mathbf{r}$-homogeneous function of degree 1 , and there exist $\underline{\sigma}, \bar{\sigma} \in \mathcal{K}_{\infty}$ such that

$$
\underline{\sigma}\left(\|x\|_{r}\right) \leq\|x\| \leq \bar{\sigma}\left(\|x\|_{r}\right) \quad \forall x \in \mathbb{R}^{n} .
$$

Consequently, due to this equivalence of the norms $\|\cdot\|$ and $\|\cdot\|_{r}$, the homogeneous norm can be used in Definition 1 . A variant of selection of $\underline{\sigma}, \bar{\sigma}$ for a particular case considered in this work is given below:

Proposition 1. If the restrictions $r_{\max } \leq 1$ and $\rho \geq 1$ are satisfied, then

$$
\begin{gathered}
\underline{\sigma}^{-1}(s)=n^{\frac{1}{\rho}}\left\{\begin{array}{ll}
s^{\frac{1}{r_{\max }}} & s \leq 1 \\
s^{\frac{1}{r_{\min }}} & s>1
\end{array},\right. \\
\bar{\sigma}(s)=n^{\rho-\frac{1}{2}}\left\{\begin{array}{ll}
s^{r_{\min }} & s \leq 1 \\
s^{r_{\max }} & s>1
\end{array} .\right.
\end{gathered}
$$

Proof: Under introduced restrictions

$$
\frac{\rho}{r_{i}} \geq 1 \quad \forall i=\overline{1, n}
$$

Using Jensen's inequality for $\rho \geq 1$ and any $a_{i} \in \mathbb{R}_{+}$for all $i=\overline{1, n}$, since the power function is concave, we obtain:

$$
\left(\sum_{i=1}^{n} a_{i}\right)^{\frac{1}{\rho}}=n^{\frac{1}{\rho}}\left(\frac{\sum_{i=1}^{n} a_{i}}{n}\right)^{\frac{1}{\rho}} \geq n^{\frac{1}{\rho}-1}\left(\sum_{i=1}^{n} a_{i}^{\frac{1}{\rho}}\right)
$$

from which the following inequality is satisfied:

$$
\|x\|_{r}=\left(\sum_{i=1}^{n}\left|x_{i}\right|^{\frac{\rho}{r_{i}}}\right) \frac{1}{\rho} \geq n^{\frac{1}{\rho}-1} \sum_{i=1}^{n}\left|x_{i}\right|^{\frac{1}{r_{i}}} \geq n^{\frac{1}{\rho}-1}\left|x_{i}\right|^{\frac{1}{r_{i}}} .
$$

Therefore,

$$
\begin{aligned}
\|x\| & =\sqrt{\sum_{i=1}^{n}\left|x_{i}\right|^{2}} \leq n^{\rho-1} \sqrt{\sum_{i=1}^{n}\|x\|_{r}^{2 r_{i}}} \\
& \leq n^{\rho-\frac{1}{2}} \begin{cases}\|x\|_{r}^{r_{\min }} & \|x\|_{r} \leq 1 \\
\|x\|_{r}^{r_{\max }} & \|x\|_{r}>1\end{cases}
\end{aligned}
$$

and we derived the desired expression of $\bar{\sigma}$. Similarly

$$
\begin{aligned}
\|x\|_{r} & =\left(\sum_{i=1}^{n}\left|x_{i}\right|^{\frac{\rho}{r_{i}}}\right)^{\frac{1}{\rho}} \leq\left(\sum_{i=1}^{n}\|x\|^{\frac{\rho}{r_{i}}}\right)^{\frac{1}{\rho}} \\
& \leq n^{\frac{1}{\rho}}\left\{\begin{array}{ll}
\|x\|^{\frac{1}{r_{\max }}} & \|x\| \leq 1 \\
\|x\|^{\frac{1}{r_{\min }}} & \|x\|>1
\end{array},\right.
\end{aligned}
$$

and inverting the above inequality we have an expression of $\underline{\sigma}$.

If system (1) is $\mathbf{r}$-homogeneous with degree $\nu$, then a direct computation shows that it is also $\tilde{\mathbf{r}}$-homogeneous for $\tilde{\mathbf{r}}=r_{\max }^{-1}\left(r_{1}, \ldots, r_{n}\right)^{T}$ with degree $\tilde{\nu}=\frac{\nu}{r_{\max }}$. Therefore, the conditions of Proposition 1 can be always satisfied for any homogeneous system with $r_{\max }=\rho=1$.

Corollary 1. Let $r_{\max } \leq 1$ and $\rho \geq 1$, then $\|\cdot\|_{r}$ is locally Lipschitz continuous.

Proof: The result is a direct consequence of (2) and the fact that the power function $x \mapsto x^{\alpha}$ for $\alpha \geq 1$ and the norm function $\|\cdot\|$ are locally Lipschitz continuous (a composition of Lipschitz continuous functions inherits the same property).

Further in this work we will always assume without loosing generality that the conditions $r_{\max } \leq 1$ and $\rho \geq 1$ are satisfied in order to use the established above continuity property of the homogeneous norm $\|\cdot\|_{r}$. 


\section{Time of convergence in homogeneous systems}

An important advantage of homogeneous systems is that their rate of convergence can be evaluated qualitatively based on their degree [24]:

Theorem 1. If (1) is $\mathbf{r}$-homogeneous of degree $\nu$ and asymptotically stable at the origin, then it is

(i) globally finite-time stable at the origin if $\nu<0$;

(ii) globally exponentially stable at the origin if $\nu=0$;

(iii) globally fixed-time stable with respect to the unit ball $B_{r}(1)$ if $\nu>0$.

Following that, we can further quantify the system convergence using the definition below.

Definition 3. For $q>1$ define $T_{q}: \mathbb{R}^{n} \rightarrow \mathbb{R}_{+}$such that

$$
\left\|X\left(T_{q}\left(x_{0}\right), x_{0}\right)\right\|_{r}=q^{-1}\left\|x_{0}\right\|_{r}
$$

i.e. it is the function of contraction in q times.

From this definition, it is easy to show that $T_{q}$ is $\mathbf{r}$-homogeneous with degree $-\nu$ (the degree of homogeneity for (1)):

$$
T_{q}\left(\Lambda_{r}(\lambda) x_{0}\right)=\lambda^{-\nu} T_{q}\left(x_{0}\right), \quad \forall x_{0} \in \mathbb{R}^{n}, \forall \lambda>0,
$$

and $T_{q}\left(x_{0}\right)$ is uniquely defined for each $x_{0}$, but it can be set-valued.

For a $\mathbf{r}$-homogeneous system (1) of degree $\nu$, if it is asymptotically stable at the origin, then there exists a continuously differentiable and $\mathbf{r}$-homogeneous function $V: \mathbb{R}^{n} \rightarrow \mathbb{R}_{+}$of degree $\mu>-\nu$ such that for all $x \in \mathbb{R}^{n}$ :

$$
\begin{gathered}
a\|x\|_{r}^{\mu} \leq V(x) \leq b\|x\|_{r}^{\mu}, \\
D^{+} V(x) f(x) \leq-c V^{1+\frac{\nu}{\mu}}(x),
\end{gathered}
$$

for some $0<a \leq b$ and $c>0$ [33], [29]. Then for any $x_{0} \in \mathbb{R}^{n}$,

$$
\left\|X\left(t, x_{0}\right)\right\|_{r} \leq \beta\left(\left\|x_{0}\right\|_{r}, t\right) \quad \forall t \geq 0
$$

where

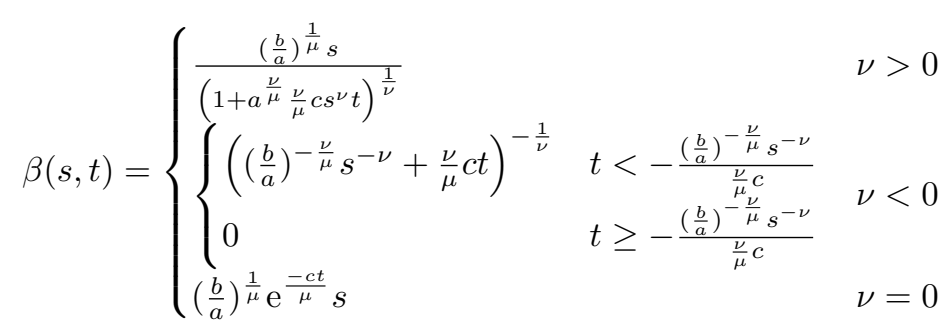

is a function from class $\mathcal{K} \mathcal{L}$, and it represents a generic parameterization of the upper bound $\beta\left(\left\|x_{0}\right\|_{r}, t\right)$ of $\left\|X\left(t, x_{0}\right)\right\|_{r}$ for all $t \geq 0$ in homogeneous systems.

Definition 4. For $q>1$, a continuous function $\bar{T}_{q}: \mathbb{R}^{n} \rightarrow \mathbb{R}_{+}$such that $\left\|X\left(t, x_{0}\right)\right\|_{r} \leq q^{-1}\left\|x_{0}\right\|_{r}$ for all $t \geq \bar{T}_{q}\left(x_{0}\right)$ for any $x_{0} \in \mathbb{R}^{n}$ is called an upper bound function of contraction in $q$ times.

Taking into account the expression of $\beta$ given in (5), we can conclude that a possible selection is $\bar{T}_{q}\left(x_{0}\right)=\bar{T}_{q}\left(\left\|x_{0}\right\|_{r}\right)$ with

$$
\text { for } s>0, \quad \bar{T}_{q}(s)=\left\{\begin{array}{ll}
\frac{q^{\nu}\left(\frac{b}{a}\right)^{\frac{\nu}{\mu}}-1}{a^{\frac{\nu}{\mu}} \frac{\nu}{\mu} c} s^{-\nu} & \nu>0 \\
\frac{\left(\frac{b}{a}\right)^{-\frac{\nu}{\mu}}-q^{\nu}}{-\frac{\nu}{\mu} c} s^{-\nu} & \nu<0 \\
\frac{\mu}{c} \ln \left(q\left(\frac{b}{a}\right)^{\frac{1}{\mu}}\right) & \nu=0
\end{array},\right.
$$

which is uniform on the spheres and skipping some ambiguity, the same symbol $\bar{T}_{q}$ is used for compactness of notation. Then $\bar{T}_{q}\left(x_{0}\right)$ can also be considered $\mathbf{r}$-homogeneous with degree $-\nu$ :

$$
\bar{T}_{q}\left(\Lambda_{r}(\lambda) x_{0}\right)=\lambda^{-\nu} \bar{T}_{q}\left(x_{0}\right), \forall x_{0} \in \mathbb{R}^{n}, \forall \lambda>0 .
$$




\section{ON HOMOGENEOUS PERTURBATIONS OF HOMOGENEOUS LYAPUNOV FUNCTIONS}

This section is devoted to the analysis of the influence of homogeneous additive perturbations on homogeneous Lyapunov functions, and the establishment of conditions providing that such a perturbation leads to another homogeneous Lyapunov function.

To this end, let us assume that for a $\mathbf{r}$-homogeneous system (1) of degree $\nu$, there exists a locally Lipschitz continuous and $\mathbf{r}$-homogeneous function $V: \mathbb{R}^{n} \rightarrow \mathbb{R}_{+}$of degree $\mu>-\nu$, then the analysis and the verification of all properties of $V$ can be performed on the sphere $S_{r}(1)$ only. Indeed, for any $x \in \mathbb{R}^{n}$ there is $y \in S_{r}(1)$ such that $x=\Lambda_{r}\left(\|x\|_{r}\right) y$, then

$$
a\|x\|_{r}^{\mu} \leq V(x)=\|x\|_{r}^{\mu} V(y) \leq b\|x\|_{r}^{\mu},
$$

where $a=\inf _{y \in S_{r}(1)} V(y)$ and $b=\sup _{y \in S_{r}(1)} V(y)$ (same as in (3)), and

$$
D^{+} V(x) f(x)=\|x\|_{r}^{\nu+\mu} D^{+} V(y) f(y) \leq-d\|x\|_{r}^{\nu+\mu}
$$

where $d=-\inf _{y \in S_{r}(1)} D^{+} V(y) f(y)\left(c=b^{-1-\frac{\nu}{\mu}} d\right.$ in (4)).

Now, for such a function $V$ let us show that there is a family of locally Lipschitz continuous and $\mathbf{r}$-homogeneous Lyapunov functions $V^{\prime}: \mathbb{R}^{n} \rightarrow \mathbb{R}_{+}$of degree $\mu$ sufficiently close to $V$. To this end, denote $\varepsilon(x)=V^{\prime}(x)-V(x)$ as the difference between these functions. Clearly $\varepsilon$ is a $\mathbf{r}$-homogeneous function of degree $\mu$. We wish to formulate the restrictions on $\varepsilon$ so that $V^{\prime}(x)=V(x)+\varepsilon(x)$ would be another Lyapunov function for (1) inheriting the properties of $V$.

Proposition 2. For a locally Lipschitz continuous and $\mathbf{r}-$ homogeneous function $V: \mathbb{R}^{n} \rightarrow \mathbb{R}_{+}$of degree $\mu>-\nu$, assume that the estimates (3), (7) are satisfied for constants $0<a \leq b$ and $d>0$. Let $\varepsilon: \mathbb{R}^{n} \rightarrow \mathbb{R}$ be a locally Lipschitz continuous and $\mathbf{r}$-homogeneous function of degree $\mu$ such that

$$
-a<\underline{\varepsilon}, \quad d>\bar{\varepsilon},
$$

where $\underline{\varepsilon}=\inf _{y \in S_{r}(1)} \varepsilon(y)$ and $\bar{\varepsilon}=\sup _{y \in S_{r}(1)} D^{+} \varepsilon(y) f(y)$. Then $V^{\prime}(x)=V(x)+\varepsilon(x)$ is a locally Lipschitz continuous and $\mathbf{r}$-homogeneous Lyapunov function for (1).

Proof: Obviously, $V^{\prime}$ is locally Lipschitz continuous and $\mathbf{r}$-homogeneous as a sum of two functions possessing these properties. Next again, for any $x \in \mathbb{R}^{n}$ there is $y \in S_{r}(1)$ such that $x=\Lambda_{r}\left(\|x\|_{r}\right) y$. By definition and by homogeneity:

$$
V^{\prime}(x)=\|x\|_{r}^{\mu} V^{\prime}(y)=\|x\|_{r}^{\mu}(V(y)+\varepsilon(y))
$$

and there exist $a^{\prime}=\inf _{y \in S_{r}(1)} V^{\prime}(y)$ and $b^{\prime}=\sup _{y \in S_{r}(1)} V^{\prime}(y)$. If

$$
0<a+\underline{\varepsilon}
$$

then the relations $0<a^{\prime} \leq b^{\prime}$ are satisfied, and for all $x \in \mathbb{R}^{n}$

$$
a^{\prime}\|x\|_{r}^{\mu} \leq V^{\prime}(x) \leq b^{\prime}\|x\|_{r}^{\mu}
$$

Similarly,

$$
\begin{aligned}
D^{+} V^{\prime}(x) f(x) & =\|x\|_{r}^{\nu+\mu} D^{+} V^{\prime}(y) f(y) \\
& =\|x\|_{r}^{\nu+\mu}\left(D^{+} V(y) f(y)+D^{+} \varepsilon(y) f(y)\right)
\end{aligned}
$$

and there exists $d^{\prime}=-\inf _{y \in S_{r}(1)} D^{+} V^{\prime}(y) f(y)$. If

$$
-\inf _{y \in S_{r}(1)} D^{+} V(y) f(y)=d>\bar{\varepsilon}=\sup _{y \in S_{r}(1)} D^{+} \varepsilon(y) f(y),
$$

then $d^{\prime}>0$.

Remark 1. To demonstrate that the restriction $d>\bar{\varepsilon}$ is always feasible for some $\varepsilon$, an upper estimate of $\bar{\varepsilon}$ can be used:

$$
\hat{\bar{\varepsilon}}=\sup _{y \in S_{r}(1)}\left\|\partial^{+} \varepsilon(y)\right\| \sup _{y \in S_{r}(1)}\|f(y)\|,
$$

where $\partial^{+} \varepsilon(y)$ is the generalized gradient defined in [9]. Then for any $f$ in (1), by minimizing the values of $\varepsilon(y)$ and $\left\|\partial^{+} \varepsilon(y)\right\|$, it is possible to ensure that all conditions of Proposition 2 are satisfied. 


\section{Constructing homogeneous Lyapunov Functions}

In this section several explicit formulas for locally Lipschitz continuous and homogeneous functions are proposed completing the results of [33], [29], where only the existence of smooth homogeneous Lyapunov functions has been substantiated without an expression that can be used for numerical synthesis. For this purpose, in this section we will assume that a homogeneous system (1) is asymptotically stable and the estimate (5) is given.

Assumption 1. Let (1) be asymptotically stable at the origin with a locally Lipschitz continuous and $\mathbf{r}-$ homogeneous vector field $f$ of degree $\nu \geq 0$.

A possible expression for the function $\beta \in \mathcal{K} \mathcal{L}$ is given just below (5) and the parameters of the function $\beta$, i.e. $a, b, c$ and $\mu$, are assumed to be fixed (as usual for the converse results). It is worth to stress that for the analysis in the sequel, these parameters are not related with a Lyapunov function of (1), they come from a possible parametrization of $\beta$ given after (5).

Remark 2. Recall that under Assumption 1, if the system (1) is locally Lipschitz continuous, then for any compact set of initial conditions $\mathcal{E} \subset \mathbb{R}^{n}$ and any time $0 \leqslant T<+\infty$, there exists $K_{\mathcal{E}, T} \in \mathbb{R}_{+}$such that [18] (assuming that the solutions originated in $\mathcal{E}$ are defined on $[0, T]$ )

$$
\left\|X\left(t, x_{1}\right)-X\left(t, x_{2}\right)\right\| \leqslant K_{\mathcal{E}, T}\left\|x_{1}-x_{2}\right\|
$$

for all $0 \leqslant t \leqslant T$ and all $x_{1}, x_{2} \in \mathcal{E}$. Using the Lipschitz continuity of $\|\cdot\|_{r}$ established in Corollary 1 and the fact that for an asymptotically stable system $X\left(t, x_{0}\right) \in \widetilde{\mathcal{E}}$ for all $t \geq 0$ and $x_{0} \in \mathcal{E}$ for some compact $\widetilde{\mathcal{E}} \subset \mathbb{R}^{n}\left(\widetilde{\mathcal{E}}=B_{r}\left(\left(\frac{b}{a}\right)^{\frac{1}{\mu}}\right)\right.$ for $\mathcal{E}=B_{r}(1)$ according to (5)), then there exists $L_{\mathcal{E}, T} \in \mathbb{R}_{+}$such that

$$
\left|\left\|X\left(t, x_{1}\right)\right\|_{r}-\left\|X\left(t, x_{2}\right)\right\|_{r}\right| \leqslant L_{\mathcal{E}, T}\left\|x_{1}-x_{2}\right\|
$$

for all $0 \leqslant t \leqslant T$ and any $x_{1}, x_{2} \in \mathcal{E}$. These constants $K_{\mathcal{E}, T}$ and $L_{\mathcal{E}, T}$ will be used below in the proofs.

\section{A. Design based on supremum of trajectories}

First, the converse Lyapunov function method initiated in the works of [26], [32] is used, which is developed below for the needs of homogeneous systems.

Lemma 1. Let Assumption 1 be satisfied. Consider

$$
V(x)=\sup _{t \geqslant 0}\left\{v(X(t, x)) k\left(v(x)^{\nu} t\right)\right\}, v(x)=\sup _{t \geqslant 0}\|X(t, x)\|_{r},
$$

where $k: \mathbb{R}_{+} \rightarrow \mathbb{R}_{+}$is a continuously differentiable function satisfying $0<\kappa_{1} \leqslant k(t) \leqslant \kappa_{2}<+\infty$ with a monotonically decreasing function $\dot{k}(t)>0$ such that $\dot{k}(t) t \leq \kappa_{3}<+\infty$ for all $t \geqslant 0$. Then $V$ is a locally Lipschitz continuous and $\mathbf{r}-$ homogeneous of degree 1 function satisfying

$$
\begin{gathered}
\kappa_{1}\|x\|_{r} \leqslant V(x) \quad \text { for all } x \in \mathbb{R}^{n}, \\
D^{+} V(x) f(x)<0 \quad \text { for a.a. } x \in \mathbb{R}^{n} \backslash\{0\} .
\end{gathered}
$$

An example of the function $k(t)$ needed in this lemma is

$$
k(t)=\left(\kappa_{1}+\kappa_{2} t\right)(1+t)^{-1}, \dot{k}(t)=\left(\kappa_{2}-\kappa_{1}\right)(1+t)^{-2}, \kappa_{2}>\kappa_{1}>0 .
$$

Proof: For any $x_{0} \in \mathbb{R}^{n}$, define

$$
v\left(x_{0}\right)=\sup _{t \geqslant 0}\left\|X\left(t, x_{0}\right)\right\|_{r},
$$

by construction $\left\|x_{0}\right\|_{r} \leqslant v\left(x_{0}\right)$ and $v(0)=0$. From the attractivity of the origin and the continuity of $X(t, \cdot)$, for any $x_{0} \in \mathbb{R}^{n}$ there exists a finite time $T_{x_{0}} \in \mathbb{R}_{+}$such that $v\left(x_{0}\right)=\sup _{0 \leqslant t \leqslant T_{x_{0}}}\left\|X\left(t, x_{0}\right)\right\|_{r}$. For a homogeneous system (1), a useful and simple choice is $T_{x_{0}}=T_{q}\left(x_{0}\right)$ for some properly selected $q>1$ (the function of contraction in $q$ times from Definition 3). To analyze continuity of the function $v$, consider

$$
\begin{aligned}
\left|v\left(x_{1}\right)-v\left(x_{2}\right)\right| & =\left|\sup _{t \geqslant 0}\left\|X\left(t, x_{1}\right)\right\|_{r}-\sup _{t \geqslant 0}\left\|X\left(t, x_{2}\right)\right\|_{r}\right| \\
& =\left|\sup _{0 \leqslant t \leqslant T_{x_{1}}}\left\|X\left(t, x_{1}\right)\right\|_{r}-\sup _{0 \leqslant t \leqslant T_{x_{2}}}\right| X\left(t, x_{2}\right) \|_{r} \mid \\
& \leqslant \sup _{0 \leqslant t \leqslant T}\left|\left\|X\left(t, x_{1}\right)\right\|_{r}-\left\|X\left(t, x_{2}\right)\right\|_{r}\right|,
\end{aligned}
$$


where $T=\max \left\{T_{x_{1}}, T_{x_{2}}\right\}$ and $x_{1}, x_{2} \in \mathbb{R}^{n}$. For any compact $\mathcal{E} \subset \mathbb{R}^{n}$ there exists $T_{\mathcal{E}}=\sup _{x_{0} \in \mathcal{E}} T_{x_{0}}$ with the property $T_{\mathcal{E}}<+\infty$. Keeping this in mind, and Lipschitz continuity of the system (1) (Remark 2), we see that

$$
\begin{aligned}
\left|v\left(x_{1}\right)-v\left(x_{2}\right)\right| & \leqslant \sup _{0 \leqslant t \leqslant T_{\mathcal{E}}}|| X\left(t, x_{1}\right)\left\|_{r}-\right\| X\left(t, x_{2}\right) \|_{r} \mid \\
& \leqslant L_{\mathcal{E}, T_{\mathcal{E}}}\left\|x_{1}-x_{2}\right\|
\end{aligned}
$$

for all $x_{1}, x_{2} \in \mathcal{E}$, and the function $v$ is Lipschitz continuous on the set $\mathcal{E}$, and locally Lipschitz continuous in $\mathbb{R}^{n}$ as needed.

Moreover, the function $v$ is not increasing on any trajectory of the system (1), indeed for any $x_{0} \in \mathbb{R}^{n}$ :

$$
\begin{aligned}
v\left(X\left(t, x_{0}\right)\right) & =\sup _{\tau \geqslant 0}\left\|X\left(\tau, X\left(t, x_{0}\right)\right)\right\|_{r}=\sup _{\tau \geqslant t}\left\|X\left(\tau, x_{0}\right)\right\|_{r} \\
& \leqslant \sup _{\tau \geqslant 0}\left\|X\left(\tau, x_{0}\right)\right\|_{r}=v\left(x_{0}\right) .
\end{aligned}
$$

In addition, $v$ is $\mathbf{r}$-homogeneous of degree 1 since for all $x_{0} \in \mathbb{R}^{n}$ and $\lambda>0$ :

$$
\begin{aligned}
v\left(\Lambda_{r}(\lambda) x_{0}\right) & =\sup _{t \geqslant 0}\left\|X\left(t, \Lambda_{r}(\lambda) x_{0}\right)\right\|_{r} \\
& =\sup _{t \geqslant 0}\left\|\Lambda_{r}(\lambda) X\left(\lambda^{\nu} t, x_{0}\right)\right\|_{r} \\
& =\lambda \sup _{t \geqslant 0}\left\|X\left(\lambda^{\nu} t, x_{0}\right)\right\|_{r} \\
& =\lambda \sup _{\tau \geqslant 0}\left\|X\left(\tau, x_{0}\right)\right\|_{r}=\lambda v\left(x_{0}\right),
\end{aligned}
$$

where the change of variables $\tau=\lambda^{\nu} t$ was used.

Now, define a new function for all $x_{0} \in \mathbb{R}^{n}$ :

$$
V\left(x_{0}\right)=\sup _{t \geqslant 0}\left\{v\left(X\left(t, x_{0}\right)\right) k\left(v\left(x_{0}\right)^{\nu} t\right)\right\},
$$

where $k: \mathbb{R}_{+} \rightarrow \mathbb{R}_{+}$is a continuously differentiable function satisfying the conditions given in the formulation of lemma. The function $V$ has a lower bound $\kappa_{1}\left\|x_{0}\right\|_{r} \leqslant V\left(x_{0}\right)$ and $V(0)=0$. In addition, for all $x_{0} \in \mathbb{R}^{n}$ and $\lambda>0$ :

$$
\begin{aligned}
V\left(\Lambda_{r}(\lambda) x_{0}\right) & =\sup _{t \geqslant 0}\left\{v\left(X\left(t, \Lambda_{r}(\lambda) x_{0}\right)\right) k\left(v\left(\Lambda_{r}(\lambda) x_{0}\right)^{\nu} t\right)\right\} \\
& =\sup _{t \geqslant 0}\left\{v\left(\Lambda_{r}(\lambda) X\left(\lambda^{\nu} t, x_{0}\right)\right) k\left(\lambda^{\nu} v\left(x_{0}\right)^{\nu} t\right)\right\} \\
& =\lambda \sup _{t \geqslant 0}\left\{v\left(X\left(\lambda^{\nu} t, x_{0}\right)\right) k\left(\lambda^{\nu} v\left(x_{0}\right)^{\nu} t\right)\right\} \\
& =\lambda \sup _{\tau \geqslant 0}\left\{v\left(X\left(\tau, x_{0}\right)\right) k\left(v\left(x_{0}\right)^{\nu} \tau\right)\right\}=\lambda V\left(x_{0}\right),
\end{aligned}
$$

where the substitution $\tau=\lambda^{\nu} t$ has been used again, and $V$ is $\mathbf{r}$-homogeneous of degree 1 . Next, for any $0<\iota_{1} \leq \iota_{2}<+\infty$ and any $x_{1}, x_{2} \in \mathcal{S}=\left\{x \in \mathbb{R}^{n}: \iota_{1} \leq v(x) \leq \iota_{2}\right\}$ consider

$$
\begin{aligned}
\left|V\left(x_{1}\right)-V\left(x_{2}\right)\right|= & \left|\sup _{t \geqslant 0}\left\{v\left(X\left(t, x_{1}\right)\right) k\left(v\left(x_{1}\right)^{\nu} t\right)\right\}-\sup _{t \geqslant 0}\left\{v\left(X\left(t, x_{2}\right)\right) k\left(v\left(x_{2}\right)^{\nu} t\right)\right\}\right| \\
= & \mid \sup _{t \geqslant 0}\left\{v\left(X\left(t, x_{1}\right)\right) k\left(v\left(x_{1}\right)^{\nu} t\right)\right\}-\sup _{t \geqslant 0}\left\{v\left(X\left(t, x_{2}\right)\right) k\left(v\left(x_{1}\right)^{\nu} t\right)\right. \\
& -\sup _{t \geqslant 0}\left\{v\left(X\left(t, x_{2}\right)\right) k\left(v\left(x_{2}\right)^{\nu} t\right)+\sup _{t \geqslant 0}\left\{v\left(X\left(t, x_{2}\right)\right) k\left(v\left(x_{1}\right)^{\nu} t\right)\right\} \mid\right. \\
\leq \quad & \quad \sup _{t \geqslant 0}\left\{v\left(X\left(t, x_{1}\right)\right) k\left(v\left(x_{1}\right)^{\nu} t\right)\right\}-\sup _{t \geqslant 0}\left\{v\left(X\left(t, x_{2}\right)\right) k\left(v\left(x_{1}\right)^{\nu} t\right)\right\} \mid \\
& +\mid \sup _{t \geqslant 0}\left\{v\left(X\left(t, x_{2}\right)\right) k\left(v\left(x_{1}\right)^{\nu} t\right)\right\}-\sup _{t \geqslant 0}\left\{v\left(X\left(t, x_{2}\right)\right) k\left(v\left(x_{2}\right)^{\nu} t\right) \mid\right. \\
\leqslant & \sup _{t \geqslant 0}\left|k\left(v\left(x_{1}\right)^{\nu} t\right)\left[v\left(X\left(t, x_{1}\right)\right)-v\left(X\left(t, x_{2}\right)\right)\right]\right| \\
& +\sup _{t \geqslant 0}\left|v\left(X\left(t, x_{2}\right)\right)\left[k\left(v\left(x_{1}\right)^{\nu} t\right)-k\left(v\left(x_{2}\right)^{\nu} t\right)\right]\right| \\
\leqslant & \kappa_{2} \sup _{t \geqslant 0}\left|v\left(X\left(t, x_{1}\right)\right)-v\left(X\left(t, x_{2}\right)\right)\right|+\iota_{2} \sup _{t \geqslant 0}\left|k\left(v\left(x_{1}\right)^{\nu} t\right)-k\left(v\left(x_{2}\right)^{\nu} t\right)\right| .
\end{aligned}
$$

For any $x_{1}, x_{2} \in \mathcal{S}$, there is $0<T<+\infty$ such that

$$
\sup _{t \geqslant 0}\left|v\left(X\left(t, x_{1}\right)\right)-v\left(X\left(t, x_{2}\right)\right)\right|=\sup _{0 \leqslant t \leqslant T}\left|v\left(X\left(t, x_{1}\right)\right)-v\left(X\left(t, x_{2}\right)\right)\right| .
$$


This property follows from the fact that $v$ is not increasing and that $v\left(X\left(+\infty, x_{1}\right)\right)=v\left(X\left(+\infty, x_{2}\right)\right)=0$ for an asymptotically stable system (1). Next, using the Mean value theorem

$$
\sup _{t \geqslant 0}\left|k\left(v\left(x_{1}\right)^{\nu} t\right)-k\left(v\left(x_{2}\right)^{\nu} t\right)\right|=\sup _{t \geqslant 0}\left|\dot{k}(\theta t) t\left(v\left(x_{1}\right)^{\nu}-v\left(x_{2}\right)^{\nu}\right)\right|,
$$

where $\theta \in\left[v\left(x_{1}\right)^{\nu}, v\left(x_{2}\right)^{\nu}\right] \subset\left[\iota_{1}^{\nu}, \iota_{2}^{\nu}\right]$, then

$$
\begin{gathered}
\sup _{t \geqslant 0}\left|k\left(v\left(x_{1}\right)^{\nu} t\right)-k\left(v\left(x_{2}\right)^{\nu} t\right)\right| \leq \kappa_{3} \iota_{1}^{-\nu}\left|v\left(x_{1}\right)^{\nu}-v\left(x_{2}\right)^{\nu}\right| \\
\leq \kappa_{4}\left\|x_{1}-x_{2}\right\|
\end{gathered}
$$

and the existence of $\kappa_{4}>0$ follows from the fact that $v$ is locally Lipschitz, $\nu \geq 0$ and $\min \left\{v\left(x_{1}\right), v\left(x_{2}\right)\right\} \geq \iota_{1}>0$. Therefore,

$$
\begin{aligned}
\left|V\left(x_{1}\right)-V\left(x_{2}\right)\right| \leqslant & \kappa_{2} \sup _{0 \leqslant t \leqslant T}\left|v\left(X\left(t, x_{1}\right)\right)-v\left(X\left(t, x_{2}\right)\right)\right|+\kappa_{4}\left\|x_{1}-x_{2}\right\| \\
\leqslant & \kappa_{2} L_{\mathcal{S}, T}\left\|X\left(t, x_{1}\right)-X\left(t, x_{2}\right)\right\|+\kappa_{4}\left\|x_{1}-x_{2}\right\| \\
& \leqslant\left(\kappa_{2} L_{\mathcal{S}, T} K_{\mathcal{S}, T}+\kappa_{4}\right)\left\|x_{1}-x_{2}\right\|
\end{aligned}
$$

for all $x_{1}, x_{2} \in \mathcal{S}$. Therefore, the function $V$ is locally Lipschitz continuous on $\mathcal{S}$ for any $0<\iota_{1} \leq \iota_{2}<+\infty$, and by homogeneity it inherits this property for all $\mathbb{R}^{n}$. In addition, $V$ is strictly decreasing for any $x_{0} \in \mathbb{R}^{n} \backslash\{0\}$ :

$$
\begin{aligned}
V\left(X\left(t, x_{0}\right)\right) & =\sup _{\tau \geqslant 0}\left\{v\left(X\left[\tau, X\left(t, x_{0}\right)\right]\right) k\left(v\left(X\left(t, x_{0}\right)\right)^{\nu} \tau\right)\right\} \\
& =\sup _{\tau \geqslant t}\left\{v\left(X\left[\tau, x_{0}\right]\right) k\left[v\left(X\left(t, x_{0}\right)\right)^{\nu}(\tau-t)\right]\right\} \\
& <\sup _{\tau \geqslant 0}\left\{v\left(X\left[\tau, x_{0}\right]\right) k\left(v\left(x_{0}\right)^{\nu} \tau\right)\right\}=V\left(x_{0}\right),
\end{aligned}
$$

where on the last step the facts, that the function $v$ is not increasing and $k$ is strictly increasing, have been used.

Denote $V(t)=V\left(X\left(t, x_{0}\right)\right)$ for any $x_{0} \in \mathbb{R}^{n}$ and $t \in \mathbb{R}_{+}$, then $\dot{V}^{+}(t)=D^{+} V\left(X\left(t, x_{0}\right)\right) f\left(X\left(t, x_{0}\right)\right)$ almost everywhere and

$$
\dot{V}^{+}(t):=\lim _{h \rightarrow 0^{+}} \sup ^{-1}\left[V\left(X\left(h, x_{0}\right)\right)-V\left(x_{0}\right)\right],
$$

then $\dot{V}^{+}(t)<0$ (since it has been established above that $\left.V\left(X\left(t, x_{0}\right)\right)<V\left(x_{0}\right)\right)$ and, hence, $D^{+} V\left(x_{0}\right) f\left(x_{0}\right)<0$ for almost all $x_{0} \in \mathbb{R}^{n} \backslash\{0\}$.

The drawback of the above construction of $v$ and $V$ is that for their calculation we have to know the solutions $X\left(t, x_{0}\right)$ for all $t \geq 0$, which is an obstruction for application. Using homogeneity once more, this shortage can be avoided:

Lemma 2. Let Assumption 1 be satisfied. Then

$$
V(x)=\sup _{0 \leqslant t \leqslant \bar{T}_{q}(x)}\left\{v(X(t, x)) k\left(v(x)^{\nu} t\right)\right\}, v(x)=\sup _{0 \leqslant t \leqslant \bar{T}_{q}(x)}\|X(t, x)\|_{r},
$$

where $k: \mathbb{R}_{+} \rightarrow \mathbb{R}_{+}$is a continuously differentiable function satisfying $0<\kappa_{1} \leqslant k(t) \leqslant \kappa_{2}<\left(\frac{b}{a}\right)^{-\frac{1}{\mu}} q \kappa_{1}$ with a monotonically decreasing function $\dot{k}(t)>0$ such that $\dot{k}(t) t \leq \kappa_{3}<+\infty$ for all $t \geqslant 0$, is a locally Lipschitz continuous and $\mathbf{r}-$ homogeneous of degree 1 function such that

$$
\begin{gathered}
\kappa_{1}\|x\|_{r} \leqslant V(x) \quad \text { for all } x \in \mathbb{R}^{n}, \\
D^{+} V(x) f(x)<0 \quad \text { for a.a. } x \in \mathbb{R}^{n} \backslash\{0\} .
\end{gathered}
$$

Proof: For any $x_{0} \in \mathbb{R}^{n}$ define

$$
v\left(x_{0}\right)=\sup _{0 \leqslant t \leqslant \bar{T}_{q}\left(x_{0}\right)}\left\|X\left(t, x_{0}\right)\right\|_{r},
$$

where $\bar{T}_{q}\left(x_{0}\right)$ is the upper bound function of contraction in $q>1$ times from Definition 4 . Note that

$$
v\left(x_{0}\right)=\sup _{0 \leqslant t \leqslant \bar{T}_{q}\left(x_{0}\right)}\left\|X\left(t, x_{0}\right)\right\|_{r}=\sup _{t \geq 0}\left\|X\left(t, x_{0}\right)\right\|_{r}
$$

and the proof of all properties of the function $v$ is the same as in Lemma 1.

Now, define a new function for all $x_{0} \in \mathbb{R}^{n}$ :

$$
V\left(x_{0}\right)=\sup _{0 \leqslant t \leqslant \bar{T}_{q}\left(x_{0}\right)}\left\{v\left(X\left(t, x_{0}\right)\right) k\left(v\left(x_{0}\right)^{\nu} t\right)\right\}
$$


where $k: \mathbb{R}_{+} \rightarrow \mathbb{R}_{+}$is a continuously differentiable function satisfying the conditions of the lemma. The function $V$ has a lower bound $\kappa_{1}\left\|x_{0}\right\|_{r} \leqslant V\left(x_{0}\right)$ and $V(0)=0$. In addition, for all $x_{0} \in \mathbb{R}^{n}$ and $\lambda>0$ :

$$
\begin{aligned}
V\left(\Lambda_{r}(\lambda) x_{0}\right) & =\sup _{0 \leqslant t \leqslant \bar{T}_{q}\left(\Lambda_{r}(\lambda) x_{0}\right)}\left\{v\left(X\left(t, \Lambda_{r}(\lambda) x_{0}\right)\right) k\left(v\left(\Lambda_{r}(\lambda) x_{0}\right)^{\nu} t\right)\right\} \\
& =\sup _{0 \leqslant t \leqslant \lambda^{-\nu} \bar{T}_{q}\left(x_{0}\right)}\left\{v\left(\Lambda_{r}(\lambda) X\left(\lambda^{\nu} t, x_{0}\right)\right) k\left(\lambda^{\nu} v\left(x_{0}\right)^{\nu} t\right)\right\} \\
& =\lambda \sup _{0 \leqslant t \leqslant \lambda^{-\nu} \bar{T}_{q}\left(x_{0}\right)}\left\{v\left(X\left(\lambda^{\nu} t, x_{0}\right)\right) k\left(\lambda^{\nu} v\left(x_{0}\right)^{\nu} t\right)\right\} \\
& =\lambda \sup _{0 \leqslant \tau \leqslant \bar{T}_{q}\left(x_{0}\right)}\left\{v\left(X\left(\tau, x_{0}\right)\right) k\left(v\left(x_{0}\right)^{\nu} \tau\right)\right\}=\lambda V\left(x_{0}\right),
\end{aligned}
$$

where the substitution $\tau=\lambda^{\nu} t$ has been used again, and $V$ is $\mathbf{r}$-homogeneous of degree 1 . Next, for any $0<\iota_{1} \leq \iota_{2}<+\infty$ and any $x_{1}, x_{2} \in \mathcal{S}=\left\{x \in \mathbb{R}^{n}: \iota_{1} \leq v(x) \leq \iota_{2}\right\}$ consider

$$
\begin{aligned}
\left|V\left(x_{1}\right)-V\left(x_{2}\right)\right|= & \left|\sup _{0 \leqslant t \leqslant \bar{T}_{q}\left(x_{1}\right)}\left\{v\left(X\left(t, x_{1}\right)\right) k\left(v\left(x_{1}\right)^{\nu} t\right)\right\}-\sup _{0 \leqslant t \leqslant \bar{T}_{q}\left(x_{2}\right)}\left\{v\left(X\left(t, x_{2}\right)\right) k\left(v\left(x_{2}\right)^{\nu} t\right)\right\}\right| \\
\leq & \left|\sup _{0 \leqslant t \leqslant \bar{T}_{q}\left(x_{1}\right)}\left\{v\left(X\left(t, x_{1}\right)\right) k\left(v\left(x_{1}\right)^{\nu} t\right)\right\}-\sup _{0 \leqslant t \leqslant \bar{T}_{q}\left(x_{2}\right)}\left\{v\left(X\left(t, x_{2}\right)\right) k\left(v\left(x_{1}\right)^{\nu} t\right)\right\}\right| \\
& +\left|\sup _{0 \leqslant t \leqslant \bar{T}_{q}\left(x_{2}\right)}\left\{v\left(X\left(t, x_{2}\right)\right) k\left(v\left(x_{1}\right)^{\nu} t\right)\right\}-\sup _{0 \leqslant t \leqslant \bar{T}_{q}\left(x_{2}\right)}\left\{v\left(X\left(t, x_{2}\right)\right) k\left(v\left(x_{2}\right)^{\nu} t\right)\right\}\right| \\
\leqslant & \sup _{0 \leqslant t \leqslant T^{\prime}}\left|k\left(v\left(x_{1}\right)^{\nu} t\right)\left[v\left(X\left(t, x_{1}\right)\right)-v\left(X\left(t, x_{2}\right)\right)\right]\right| \\
& +\sup _{0 \leqslant t \leqslant \bar{T}_{q}\left(x_{2}\right)}\left|v\left(X\left(t, x_{2}\right)\right)\left\{k\left(v\left(x_{1}\right)^{\nu} t\right)-k\left(v\left(x_{2}\right)^{\nu} t\right)\right\}\right| \\
\leqslant & \kappa_{2} \sup _{0 \leqslant t \leqslant T^{\prime}}\left|v\left(X\left(t, x_{1}\right)\right)-v\left(X\left(t, x_{2}\right)\right)\right|+\iota_{2} \sup _{0 \leqslant t \leqslant T^{\prime}}\left|k\left(v\left(x_{1}\right)^{\nu} t\right)-k\left(v\left(x_{2}\right)^{\nu} t\right)\right|,
\end{aligned}
$$

where as before $T^{\prime}=\sup _{x_{0} \in \mathcal{S}} T_{q}\left(x_{0}\right)$. Recalling the properties of the function $k(t)$ and using the Mean value theorem we derive:

$$
\sup _{0 \leqslant t \leqslant T^{\prime}}\left|k\left(v\left(x_{1}\right)^{\nu} t\right)-k\left(v\left(x_{2}\right)^{\nu} t\right)\right|=\sup _{0 \leqslant t \leqslant T^{\prime}}\left|\dot{k}(\theta t) t\left(v\left(x_{1}\right)^{\nu}-v\left(x_{2}\right)^{\nu}\right)\right|
$$

for $\theta \subset\left[\iota_{1}^{\nu}, \iota_{2}^{\nu}\right]$, then

$$
\sup _{0 \leqslant t \leqslant T^{\prime}}\left|\dot{k}(\theta t) t\left(v\left(x_{1}\right)^{\nu}-v\left(x_{2}\right)^{\nu}\right)\right| \leq \kappa_{3} \iota_{1}^{-\nu}\left|v\left(x_{1}\right)^{\nu}-v\left(x_{2}\right)^{\nu}\right|
$$

and since $\iota_{1}>0$ there is $\kappa_{4}>0$ such that

$$
\kappa_{3} \iota_{1}^{-\nu}\left|v\left(x_{1}\right)^{\nu}-v\left(x_{2}\right)^{\nu}\right| \leq \kappa_{4}\left\|x_{1}-x_{2}\right\|
$$

Consequently, for a locally Lipschitz and stable system (1) we obtain (Remark 2)

$$
\begin{aligned}
\left|V\left(x_{1}\right)-V\left(x_{2}\right)\right| \leqslant & \kappa_{2} \sup _{0 \leqslant t \leqslant T^{\prime}}\left|v\left(X\left(t, x_{1}\right)\right)-v\left(X\left(t, x_{2}\right)\right)\right|+\kappa_{4}\left\|x_{1}-x_{2}\right\| \\
\leqslant & \kappa_{2} L_{\mathcal{S}, T^{\prime}}\left\|X\left(t, x_{1}\right)-X\left(t, x_{2}\right)\right\|+\kappa_{4}\left\|x_{1}-x_{2}\right\| \\
& \leqslant\left(\kappa_{2} L_{\mathcal{S}, T^{\prime}} K_{\mathcal{S}, T^{\prime}}+\kappa_{4}\right)\left\|x_{1}-x_{2}\right\|
\end{aligned}
$$

for all $x_{1}, x_{2} \in \mathcal{S}$. Therefore, the function $V$ is locally Lipschitz continuous on $\mathcal{S}$ for any $0<\iota_{1} \leq \iota_{2}<+\infty$, and by homogeneity it inherits this property for all $\mathbb{R}^{n}$. Recall that $v\left(X\left(t, x_{0}\right)\right)$ is not increasing and $k(t)$ is strictly increasing in time, then $V$ is strictly decreasing for any $x_{0} \in \mathbb{R}^{n} \backslash\{0\}$, and to demonstrate this fact note that

$$
\begin{aligned}
V\left(X\left(t, x_{0}\right)\right) & =\sup _{0 \leqslant \tau \leqslant T_{q}\left(X\left(t, x_{0}\right)\right)}\left\{v\left(X\left[\tau, X\left(t, x_{0}\right)\right]\right) k\left(v\left(X\left(t, x_{0}\right)\right)^{\nu} \tau\right)\right\} \\
& =\sup _{0 \leqslant \tau \leqslant T_{q}\left(X\left(t, x_{0}\right)\right)}\left\{v\left(X\left[\tau+t, x_{0}\right]\right) k\left(v\left(X\left(t, x_{0}\right)\right)^{\nu} \tau\right)\right\} \\
& =\sup _{t \leqslant s \leqslant t+\bar{T}_{q}\left(X\left(t, x_{0}\right)\right)}\left\{v\left(X\left[s, x_{0}\right]\right) k\left[v\left(X\left(t, x_{0}\right)\right)^{\nu}(s-t)\right]\right\} .
\end{aligned}
$$


If $t+\bar{T}_{q}\left(X\left(t, x_{0}\right)\right) \leq \bar{T}_{q}\left(x_{0}\right)$, then obviously

$$
\begin{aligned}
V\left(X\left(t, x_{0}\right)\right) & \leq \sup _{t \leqslant s \leqslant \bar{T}_{q}\left(x_{0}\right)}\left\{v\left(X\left[s, x_{0}\right]\right) k\left[v\left(X\left(t, x_{0}\right)\right)^{\nu}(s-t)\right]\right\} \\
& <\sup _{0 \leqslant s \leqslant \bar{T}_{q}\left(x_{0}\right)}\left\{v\left(X\left[s, x_{0}\right]\right) k\left[v\left(X\left(t, x_{0}\right)\right)^{\nu} s\right]\right\} \\
& \leq \sup _{0 \leqslant s \leqslant \bar{T}_{q}\left(x_{0}\right)}\left\{v\left(X\left[s, x_{0}\right]\right) k\left[v\left(x_{0}\right)^{\nu} s\right]\right\}=V\left(x_{0}\right) .
\end{aligned}
$$

If $t+\bar{T}_{q}\left(X\left(t, x_{0}\right)\right)>\bar{T}_{q}\left(x_{0}\right)$, then $\left\|X\left(t, x_{0}\right)\right\|_{r}<\left\|x_{0}\right\|_{r}$ and (if $t>\bar{T}_{q}\left(x_{0}\right)$ then the first supremum above disappears and the equality sign has to be replaced with the sign less or equal)

$$
\begin{aligned}
V\left(X\left(t, x_{0}\right)\right)= & \max \left\{\sup _{t \leqslant s \leqslant \bar{T}_{q}\left(x_{0}\right)}\left\{v\left(X\left[s, x_{0}\right]\right) k\left[v\left(X\left(t, x_{0}\right)\right)^{\nu}(s-t)\right]\right\},\right. \\
& \bar{T}_{q}\left(x_{0}\right) \leqslant s \leqslant t+\bar{T}_{q}\left(X\left(t, x_{0}\right)\right) \\
\leq & \left.\left.\max \left\{\sup _{t \leqslant s \leqslant \bar{T}_{q}\left(x_{0}\right)}\left\{v\left(X\left[s, x_{0}\right]\right) k\left[v\left(X\left(t, x_{0}\right)\right)^{\nu}(s-t)\right]\right\}\right\} k\left[v\left(X\left(t, x_{0}\right)\right)^{\nu}(s-t)\right]\right\}, \kappa_{2} v\left(X\left[\bar{T}_{q}\left(x_{0}\right), x_{0}\right]\right)\right\} \\
\leq & \max \left\{\sup _{t \leqslant s \leqslant \bar{T}_{q}\left(x_{0}\right)}\left\{v\left(X\left[s, x_{0}\right]\right) k\left[v\left(X\left(t, x_{0}\right)\right)^{\nu}(s-t)\right]\right\}, \kappa_{2}\left(\frac{b}{a}\right)^{\frac{1}{\mu}} q^{-1}\left\|x_{0}\right\|_{r}\right\} .
\end{aligned}
$$

Let $\kappa_{2}\left(\frac{b}{a}\right)^{\frac{1}{\mu}} q^{-1}<\kappa_{1}$, then

$$
\begin{aligned}
V\left(X\left(t, x_{0}\right)\right) & <\max \left\{\sup _{t \leqslant s \leqslant \bar{T}_{q}\left(x_{0}\right)}\left\{v\left(X\left[s, x_{0}\right]\right) k\left[v\left(X\left(t, x_{0}\right)\right)^{\nu}(s-t)\right]\right\}, V\left(x_{0}\right)\right\} \\
& <\max \left\{\sup _{0 \leqslant s \leqslant \bar{T}_{q}\left(x_{0}\right)}\left\{v\left(X\left[s, x_{0}\right]\right) k\left[v\left(x_{0}\right)^{\nu} s\right]\right\}, V\left(x_{0}\right)\right\}=V\left(x_{0}\right)
\end{aligned}
$$

and we derived the required property that

$$
V\left(X\left(t, x_{0}\right)\right)<V\left(x_{0}\right)
$$

for all $t>0$.

Remark 3. Note that the functions $v(x)$ given in lemmas 1 and 2 are also locally Lipschitz continuous and $\mathbf{r}$-homogeneous of degree 1 such that

$$
\begin{gathered}
\left\|x_{0}\right\|_{r} \leqslant v(x) \leqslant\left(\frac{b}{a}\right)^{\frac{1}{\mu}}\left\|x_{0}\right\|_{r} \quad \text { for all } x \in \mathbb{R}^{n}, \\
D^{+} v(x) f(x) \leqslant 0 \quad \text { for a.a. } x \in \mathbb{R}^{n} \backslash\{0\} .
\end{gathered}
$$

Thus, $v(x)$ are non-strict Lyapunov functions for (1). It is worth to note that it is difficult to make a numeric derivation of $v$ since any small computational error may lead to a function $v$ with a positive derivative along trajectories due to its non-strictness.

\section{B. Design based on integral of trajectories}

Following [23], [20], an alternative integral construction of a strict Lyapunov function can be used:

Lemma 3. Let Assumption 1 be satisfied. Then for $\mu>\max \{1, \nu\}$,

$$
W(x)=\int_{0}^{+\infty}\|X(t, x)\|_{r}^{\mu} d t
$$

is a locally Lipschitz continuous and $\mathbf{r}$-homogeneous of degree $\mu-\nu$ function such that

$$
\begin{gathered}
T_{q}^{\min } q^{-\mu}\|x\|_{r}^{\mu-\nu} \leqslant W(x), \forall x \in \mathbb{R}^{n}, \\
D^{+} W(x) f(x)<0 \quad \text { for a.a. } x \in \mathbb{R}^{n} \backslash\{0\},
\end{gathered}
$$

where $T_{q}^{\min }=\inf _{x_{0} \in S_{r}(1)} T_{q}\left(x_{0}\right)$. 
Proof: For any $x_{0} \in \mathbb{R}^{n}$ consider

$$
W\left(x_{0}\right)=\int_{0}^{+\infty}\left\|X\left(t, x_{0}\right)\right\|_{r}^{\mu} d t
$$

which is a well-defined integral for any $x_{0} \in \mathbb{R}^{n}$ under Assumption 1 for the function $\beta \in \mathcal{K} \mathcal{L}$ given below (5) (a direct computation confirms this fact). The obtained function $W$ is $\mathbf{r}$-homogeneous of degree $\mu-\nu$ since for all $x_{0} \in \mathbb{R}^{n}$ and $\lambda>0$ :

$$
\begin{aligned}
W\left(\Lambda_{r}(\lambda) x_{0}\right) & =\int_{0}^{+\infty}\left\|X\left(t, \Lambda_{r}(\lambda) x_{0}\right)\right\|_{r}^{\mu} d t \\
& =\lambda^{\mu} \int_{0}^{+\infty}\left\|X\left(\lambda^{\nu} t, x_{0}\right)\right\|_{r}^{\mu} d t \\
& =\lambda^{\mu-\nu} \int_{0}^{+\infty}\left\|X\left(\tau, x_{0}\right)\right\|_{r}^{\mu} d \tau=\lambda^{\mu-\nu} W\left(x_{0}\right),
\end{aligned}
$$

where the change of variables $\tau=\lambda^{\nu} t$ was used. By construction $W(0)=0$ and for any $x_{0} \in S_{r}(1)$ :

$$
W\left(x_{0}\right)=\int_{0}^{+\infty}\left\|X\left(t, x_{0}\right)\right\|_{r}^{\mu} d t \geq \int_{0}^{T_{q}\left(x_{0}\right)} q^{-\mu} d t=T_{q}\left(x_{0}\right) q^{-\mu},
$$

hence by homogeneity $T_{q}^{\min } q^{-\mu}\|x\|_{r}^{\mu-\nu} \leqslant W(x)$ for all $x \in \mathbb{R}^{n}$. Since $\mu>1, X\left(t, x_{0}\right)$ is locally Lipschitz continuous in $x_{0}$ for an asymptotically stable system (1) with locally Lipschitz $f$, and the Lipschitz continuity of $\|\cdot\|_{r}$ is established in Corollary 1 , then $W$ is locally Lipschitz continuous in $\mathbb{R}^{n}$ as needed.

Moreover, the function $W$ is strictly decreasing on any trajectory of the system (1), indeed for any $x_{0} \in \mathbb{R}^{n}$ :

$$
\begin{aligned}
W\left(X\left(t, x_{0}\right)\right) & =\int_{0}^{+\infty}\left\|X\left(\tau, X\left(t, x_{0}\right)\right)\right\|_{r}^{\mu} d \tau \\
& =\int_{0}^{+\infty}\left\|X\left(\tau+t, x_{0}\right)\right\|_{r}^{\mu} d \tau \\
& =\int_{t}^{+\infty}\left\|X\left(s, x_{0}\right)\right\|_{r}^{\mu} d s \\
& <\int_{0}^{+\infty}\left\|X\left(s, x_{0}\right)\right\|_{r}^{\mu} d s=W\left(x_{0}\right),
\end{aligned}
$$

where the strict sign is obtained since for any $t>0$ and $x_{0} \neq 0$ there are time instants $s \in(0, t)$ such that $\left\|X\left(s, x_{0}\right)\right\|_{r} \neq 0$.

For the case of conventional homogeneity, Lemma 3 was proven in [33] (Theorem 36). Finally, a new more practical construction on a fixed time interval might be preferable:

Theorem 2. Let Assumption 1 be satisfied. Then there exists $q>1$ such that

$$
U(x)=\int_{0}^{\bar{T}_{q}\left(\|x\|_{r}\right)}\|X(t, x)\|_{r}^{\mu} d t
$$

where $\bar{T}_{q}\left(\|x\|_{r}\right)$ is the upper bound function of contraction in q times (an example is given in (6)), for $\mu>\max \left\{1, \nu+\nu^{2}\right\}$ is a locally Lipschitz continuous and $\mathbf{r}$-homogeneous of degree $\mu-\nu$ function such that

$$
\begin{gathered}
T_{q}^{\min } q^{-\mu}\|x\|_{r}^{\mu-\nu} \leqslant U(x) \leqslant \bar{T}_{q}(1) \frac{b}{a}\|x\|_{r}^{\mu-\nu}, \forall x \in \mathbb{R}^{n}, \\
D^{+} U(x) f(x)<0 \quad \text { for a.a. } x \in \mathbb{R}^{n} \backslash\{0\},
\end{gathered}
$$

where $T_{q}^{\min }=\inf _{x \in S_{r}(1)} T_{q}(x)$.

Proof: For any $x_{0} \in \mathbb{R}^{n}$ define

$$
U\left(x_{0}\right)=\int_{0}^{\bar{T}_{q}\left(\left\|x_{0}\right\|_{r}\right)}\left\|X\left(t, x_{0}\right)\right\|_{r}^{\mu} d t
$$


where $\bar{T}_{q}\left(\left\|x_{0}\right\|_{r}\right)$ for $q>1$ is from Definition 4, which is defined as a function of $\left\|x_{0}\right\|_{r}$. The function $U$ is $\mathbf{r}$-homogeneous of degree $\mu-\nu$ since for all $x_{0} \in \mathbb{R}^{n}$ and $\lambda>0$ :

$$
\begin{aligned}
U\left(\Lambda_{r}(\lambda) x_{0}\right) & =\int_{0}^{\bar{T}_{q}\left(\left\|\Lambda_{r}(\lambda) x_{0}\right\|_{r}\right)}\left\|X\left(t, \Lambda_{r}(\lambda) x_{0}\right)\right\|_{r}^{\mu} d t \\
& =\lambda^{\mu} \int_{0}^{\lambda^{-\nu} \bar{T}_{q}\left(\left\|x_{0}\right\|_{r}\right)}\left\|X\left(\lambda^{\nu} t, x_{0}\right)\right\|_{r}^{\mu} d t \\
& =\lambda^{\mu-\nu} \int_{0}^{\bar{T}_{q}\left(\left\|x_{0}\right\|_{r}\right)}\left\|X\left(\tau, x_{0}\right)\right\|_{r}^{\mu} d \tau=\lambda^{\mu-\nu} U\left(x_{0}\right),
\end{aligned}
$$

where the change of variables $\tau=\lambda^{\nu} t$ was used. By construction $U(0)=0$ and for any $x_{0} \in S_{r}(1)$ :

$$
U\left(x_{0}\right)=\int_{0}^{\bar{T}_{q}(1)}\left\|X\left(t, x_{0}\right)\right\|_{r}^{\mu} d t \geq \int_{0}^{T_{q}\left(x_{0}\right)} q^{-\mu} d t=T_{q}\left(x_{0}\right) q^{-\mu}
$$

and similarly using (5):

$$
U\left(x_{0}\right)=\int_{0}^{\bar{T}_{q}(1)}\left\|X\left(t, x_{0}\right)\right\|_{r}^{\mu} d t \leq \int_{0}^{\bar{T}_{q}(1)} \frac{b}{a} d t=\bar{T}_{q}(1) \frac{b}{a}
$$

Hence, by homogeneity

$$
T_{q}^{\min } q^{-\mu}\|x\|_{r}^{\mu-\nu} \leqslant U(x) \leq \bar{T}_{q}(1) \frac{b}{a}\|x\|_{r}^{\mu-\nu}
$$

for all $x \in \mathbb{R}^{n}$. Further in the proof we will also need the following estimate:

$$
\begin{aligned}
U\left(x_{0}\right) & =\int_{0}^{\bar{T}_{q}(1)}\left\|X\left(t, x_{0}\right)\right\|_{r}^{\mu} d t \\
& \geq \int_{0}^{\bar{T}_{\underline{q}}(1)}\left\|X\left(t, x_{0}\right)\right\|_{r}^{\mu} d t \\
& \geq \int_{0}^{\bar{T}_{\underline{q}}(1)} \underline{q}^{-\mu} d t=\underline{q}^{-\mu} \bar{T}_{\underline{q}}(1)
\end{aligned}
$$

where $1<\underline{q}<q$ (then $\left.\bar{T}_{\underline{q}}(1)<\bar{T}_{q}(1)\right)$. Denote

$$
\kappa=\lim _{\underline{q} \rightarrow 1} \underline{q}^{-\mu} \bar{T}_{\underline{q}}(1)=\left\{\begin{array}{ll}
\frac{\left(\frac{b}{a}\right)^{\frac{\nu}{\mu}}-1}{a^{\frac{\nu}{\mu}} \frac{\nu}{\mu} c} & \nu>0 \\
\frac{\left(\frac{b}{a}\right)^{-\frac{\nu}{\mu}}-1}{-\frac{\nu}{\mu} c} & \nu<0 \\
\frac{1}{c} \ln \left(\frac{b}{a}\right) & \nu=0
\end{array} .\right.
$$

If $b>a$ (we can impose such a restriction without loosing generality), then $\kappa>0$ and by homogeneity for all $x \in \mathbb{R}^{n}$ :

$$
U(x) \geq \kappa\|x\|_{r}^{\mu-\nu} .
$$

To analyze the continuity of the function $U$, consider $x_{1}, x_{2} \in S_{r}(1)$ and

$$
\begin{aligned}
\left|U\left(x_{1}\right)-U\left(x_{2}\right)\right| & =\left|\int_{0}^{\bar{T}_{q}\left(\left\|x_{1}\right\|_{r}\right)}\left\|X\left(t, x_{1}\right)\right\|_{r}^{\mu} d t-\int_{0}^{\bar{T}_{q}\left(\left\|x_{2}\right\|_{r}\right)}\left\|X\left(t, x_{2}\right)\right\|_{r}^{\mu} d t\right| \\
& =\left|\int_{0}^{\bar{T}_{q}(1)}\left\|X\left(t, x_{1}\right)\right\|_{r}^{\mu}-\left\|X\left(t, x_{2}\right)\right\|_{r}^{\mu} d t\right| \\
& \leq \int_{0}^{\bar{T}_{q}(1)}\left|\left\|X\left(t, x_{1}\right)\right\|_{r}^{\mu}-\left\|X\left(t, x_{2}\right)\right\|_{r}^{\mu}\right| d t .
\end{aligned}
$$

Due to Lipschitz continuity of the system (1) there exists $L_{S_{r}(1), \bar{T}_{q}(1)} \in \mathbb{R}_{+}$(Remark 2) such that

$$
\left|\left\|X\left(t, x_{1}\right)\right\|_{r}^{\mu}-\left\|X\left(t, x_{2}\right)\right\|_{r}^{\mu}\right| \leqslant L_{S_{r}(1), \bar{T}_{q}(1)}\left\|x_{1}-x_{2}\right\|,
$$


for all $0 \leqslant t \leqslant \bar{T}_{q}(1)$ and any $x_{1}, x_{2} \in S_{r}(1)$. Consequently,

$$
\begin{aligned}
\left|U\left(x_{1}\right)-U\left(x_{2}\right)\right| & \leqslant \int_{0}^{\bar{T}_{q}(1)} L_{S_{r}(1), \bar{T}_{q}(1)}\left\|x_{1}-x_{2}\right\| d t \\
& \leqslant \bar{T}_{q}(1) L_{S_{r}(1), \bar{T}_{q}(1)}\left\|x_{1}-x_{2}\right\|
\end{aligned}
$$

for all $x_{1}, x_{2} \in S_{r}(1)$, and the function $U$ is Lipschitz continuous on the unit sphere $S_{r}(1)$, and by homogeneity it is locally Lipschitz continuous in $\mathbb{R}^{n}$ as needed.

Moreover, the function $U$ is decreasing on any trajectory of the system (1), indeed for any $x_{0} \in \mathbb{R}^{n}$ :

$$
\begin{aligned}
U\left(X\left(t, x_{0}\right)\right) & =\int_{0}^{\bar{T}_{q}\left(\left\|X\left(t, x_{0}\right)\right\|_{r}\right)}\left\|X\left(\tau, X\left(t, x_{0}\right)\right)\right\|_{r}^{\mu} d \tau \\
& =\int_{0}^{\bar{T}_{q}\left(\left\|X\left(t, x_{0}\right)\right\|_{r}\right)}\left\|X\left(\tau+t, x_{0}\right)\right\|_{r}^{\mu} d \tau \\
& =\int_{t}^{t+\bar{T}_{q}\left(\left\|X\left(t, x_{0}\right)\right\|_{r}\right)}\left\|X\left(s, x_{0}\right)\right\|_{r}^{\mu} d s .
\end{aligned}
$$

If $t+\bar{T}_{q}\left(\left\|X\left(t, x_{0}\right)\right\|_{r}\right) \leq \bar{T}_{q}\left(\left\|x_{0}\right\|_{r}\right)$, then

$$
\begin{aligned}
U\left(X\left(t, x_{0}\right)\right) & \leq \int_{t}^{\bar{T}_{q}\left(\left\|x_{0}\right\|_{r}\right)}\left\|X\left(s, x_{0}\right)\right\|_{r}^{\mu} d s \\
& <\int_{0}^{\bar{T}_{q}\left(\left\|x_{0}\right\|_{r}\right)}\left\|X\left(s, x_{0}\right)\right\|_{r}^{\mu} d s=U\left(x_{0}\right),
\end{aligned}
$$

where the strict sign appears since $X\left(s, x_{0}\right) \neq 0$ for $s \in[0, t]$. Consider the case $0<t<\bar{T}_{\gamma q}\left(\left\|x_{0}\right\|_{r}\right)$ where $q^{-1}<\gamma<1$ is a constant to be determined later (if for all $x_{0} \in \mathbb{R}^{n}$ and $t \in\left(0, \bar{T}_{\gamma q}\left(\left\|x_{0}\right\|_{r}\right)\right)$ the function $U$ is strictly decreasing, then it is enough for our purpose), then for $t<\bar{T}_{\gamma q}\left(\left\|x_{0}\right\|_{r}\right)$ we have

$$
\begin{aligned}
\int_{0}^{t}\left\|X\left(s, x_{0}\right)\right\|_{r}^{\mu} d s & \geq \int_{0}^{t} \gamma^{-\mu} q^{-\mu}\left\|x_{0}\right\|_{r}^{\mu} d s \\
& >\int_{0}^{t} q^{-\mu}\left\|x_{0}\right\|_{r}^{\mu} d s=q^{-\mu}\left\|x_{0}\right\|_{r}^{\mu} t
\end{aligned}
$$

Under the restriction $t+\bar{T}_{q}\left(\left\|X\left(t, x_{0}\right)\right\|_{r}\right)>\bar{T}_{q}\left(\left\|x_{0}\right\|_{r}\right)$ let $\bar{T}_{q}\left(\left\|X\left(t, x_{0}\right)\right\|_{r}\right) \leq \bar{T}_{q}\left(\left\|x_{0}\right\|_{r}\right)$ then

$$
\begin{aligned}
U\left(X\left(t, x_{0}\right)\right)-U\left(x_{0}\right) & =\int_{t}^{t+\bar{T}_{q}\left(\left\|X\left(t, x_{0}\right)\right\|_{r}\right)}\left\|X\left(s, x_{0}\right)\right\|_{r}^{\mu} d s-\int_{0}^{\bar{T}_{q}\left(\left\|x_{0}\right\|_{r}\right)}\left\|X\left(s, x_{0}\right)\right\|_{r}^{\mu} d s \\
& \leq \int_{t}^{t+\bar{T}_{q}\left(\left\|X\left(t, x_{0}\right)\right\|_{r}\right)}\left\|X\left(s, x_{0}\right)\right\|_{r}^{\mu} d s-\int_{0}^{\bar{T}_{q}\left(\left\|X\left(t, x_{0}\right)\right\|_{r}\right)}\left\|X\left(s, x_{0}\right)\right\|_{r}^{\mu} d s \\
& =\int_{\bar{T}_{q}\left(\left\|X\left(t, x_{0}\right)\right\|_{r}\right)}^{t+\bar{T}_{q}\left(\left\|X\left(t, x_{0}\right)\right\|_{r}\right)}\left\|X\left(s, x_{0}\right)\right\|_{r}^{\mu} d s-\int_{0}^{t}\left\|X\left(s, x_{0}\right)\right\|_{r}^{\mu} d s \\
& \leq \int_{\bar{T}_{q}\left(\left\|X\left(t, x_{0}\right)\right\|_{r}\right)}^{t+\bar{T}_{q}\left(\left\|X\left(t, x_{0}\right)\right\|_{r}\right)} q^{-\mu}\left\|x_{0}\right\|_{r}^{\mu} d s-\int_{0}^{t}\left\|X\left(s, x_{0}\right)\right\|_{r}^{\mu} d s \\
& =q^{-\mu}\left\|x_{0}\right\|_{r}^{\mu} t-\int_{0}^{t}\left\|X\left(s, x_{0}\right)\right\|_{r}^{\mu} d s<0 .
\end{aligned}
$$

Hence, in this case again $U\left(X\left(t, x_{0}\right)\right)<U\left(x_{0}\right)$. Finally, let us analyze the case $\bar{T}_{q}\left(\left\|X\left(t, x_{0}\right)\right\|_{r}\right)>\bar{T}_{q}\left(\left\|x_{0}\right\|_{r}\right)$. Note that if $\left\|X\left(t, x_{0}\right)\right\|_{r}<\frac{\kappa}{\bar{T}_{q}(1) \frac{b}{a}}\left\|x_{0}\right\|_{r}$ then

$$
U\left(X\left(t, x_{0}\right)\right) \leq \bar{T}_{q}(1) \frac{b}{a}\left\|X\left(t, x_{0}\right)\right\|_{r}^{\mu-\nu}<\kappa\left\|x_{0}\right\|_{r}^{\mu-\nu} \leq U\left(x_{0}\right),
$$

therefore, the case with $\left\|X\left(t, x_{0}\right)\right\|_{r} \geq \frac{\kappa}{\bar{T}_{q}(1) \frac{b}{a}}\left\|x_{0}\right\|_{r}$ has to be considered only, and due to homogeneity of $\bar{T}_{q}$, the condition $\bar{T}_{q}\left(\left\|X\left(t, x_{0}\right)\right\|_{r}\right)>\bar{T}_{q}\left(\left\|x_{0}\right\|_{r}\right)$ implies that the analysis can be even further restricted to the case 
$\frac{\kappa}{\bar{T}_{q}(1) \frac{b}{a}}\left\|x_{0}\right\|_{r} \leq\left\|X\left(t, x_{0}\right)\right\|_{r}<\left\|x_{0}\right\|_{r}$ and $\bar{T}_{q}\left(\left\|x_{0}\right\|_{r}\right)<\bar{T}_{q}\left(\left\|X\left(t, x_{0}\right)\right\|_{r}\right) \leq \varrho \bar{T}_{q}\left(\left\|x_{0}\right\|_{r}\right)$, where $\varrho=\left(\frac{\kappa}{\bar{T}_{q}(1) \frac{b}{a}}\right)^{-\nu}$. Taking into account all these restrictions we have:

$$
\begin{aligned}
U\left(X\left(t, x_{0}\right)\right)-U\left(x_{0}\right) & =\int_{t}^{t+\bar{T}_{q}\left(\left\|X\left(t, x_{0}\right)\right\|_{r}\right)}\left\|X\left(s, x_{0}\right)\right\|_{r}^{\mu} d s-\int_{0}^{\bar{T}_{q}\left(\left\|x_{0}\right\|_{r}\right)}\left\|X\left(s, x_{0}\right)\right\|_{r}^{\mu} d s \\
& =\int_{\bar{T}_{q}\left(\left\|x_{0}\right\|_{r}\right)}^{t+\bar{T}_{q}\left(\left\|X\left(t, x_{0}\right)\right\|_{r}\right)}\left\|X\left(s, x_{0}\right)\right\|_{r}^{\mu} d s-\int_{0}^{t}\left\|X\left(s, x_{0}\right)\right\|_{r}^{\mu} d s \\
& \leq \int_{\bar{T}_{q}\left(\left\|x_{0}\right\|_{r}\right)}^{t+\bar{T}_{q}\left(\left\|X\left(t, x_{0}\right)\right\|_{r}\right)} q^{-\mu}\left\|x_{0}\right\|_{r}^{\mu} d s-\int_{0}^{t}\left\|X\left(s, x_{0}\right)\right\|_{r}^{\mu} d s \\
& =q^{-\mu}\left\|x_{0}\right\|_{r}^{\mu}\left(t+\bar{T}_{q}\left(\left\|X\left(t, x_{0}\right)\right\|_{r}\right)-\bar{T}_{q}\left(\left\|x_{0}\right\|_{r}\right)\right)-\int_{0}^{t}\left\|X\left(s, x_{0}\right)\right\|_{r}^{\mu} d s \\
& \leq q^{-\mu}\left\|x_{0}\right\|_{r}^{\mu}\left(t+\bar{T}_{q}\left(\left\|X\left(t, x_{0}\right)\right\|_{r}\right)-\bar{T}_{q}\left(\left\|x_{0}\right\|_{r}\right)\right)-\gamma^{-\mu} q^{-\mu}\left\|x_{0}\right\|_{r}^{\mu} t,
\end{aligned}
$$

then

$$
\bar{T}_{q}\left(\left\|X\left(t, x_{0}\right)\right\|_{r}\right)-\bar{T}_{q}\left(\left\|x_{0}\right\|_{r}\right)<\left(\gamma^{-\mu}-1\right) t
$$

implies that $U\left(X\left(t, x_{0}\right)\right)<U\left(x_{0}\right)$. Note that

$$
\begin{gathered}
\bar{T}_{q}\left(\left\|X\left(t, x_{0}\right)\right\|_{r}\right)-\bar{T}_{q}\left(\left\|x_{0}\right\|_{r}\right)=\frac{\left\|x_{0}\right\|_{r}^{\nu}-\left\|X\left(t, x_{0}\right)\right\|_{r}^{\nu}}{\left\|X\left(t, x_{0}\right)\right\|_{r}^{\nu}} \bar{T}_{q}\left(\left\|x_{0}\right\|_{r}\right) \\
\leq \frac{\left\|x_{0}\right\|_{r}^{\nu}-\left\|X\left(t, x_{0}\right)\right\|_{r}^{\nu}}{\varrho^{-1}\left\|x_{0}\right\|_{r}^{\nu}} \bar{T}_{q}\left(\left\|x_{0}\right\|_{r}\right) .
\end{gathered}
$$

Let us consider $x_{0} \in S_{r}(1)$, the solution $X\left(t, x_{0}\right)$ is a continuously differentiable function for almost all $t \geq 0$, then for all $t \in\left(0, \bar{T}_{\gamma q}(1)\right)$ and all $x_{0} \in S_{r}(1)$ there exists $L>0$ such that $\left|\left\|x_{0}\right\|_{r}^{\nu}-\left\|X\left(t, x_{0}\right)\right\|_{r}^{\nu}\right| \leq L t$ (recall that $\nu \geq 0$ and the Lipschitz continuity of $\|\cdot\|_{r}$ is established in Corollary 1). Consequently, for $x_{0} \in S_{r}(1)$

$$
\begin{gathered}
\frac{\left\|x_{0}\right\|_{r}^{\nu}-\left\|X\left(t, x_{0}\right)\right\|_{r}^{\nu}}{\varrho^{-1}\left\|x_{0}\right\|_{r}} \bar{T}_{q}\left(\left\|x_{0}\right\|_{r}\right)=\frac{\left\|x_{0}\right\|_{r}^{\nu}-\left\|X\left(t, x_{0}\right)\right\|_{r}^{\nu}}{\varrho^{-1}} \bar{T}_{q}(1) \\
\leq \varrho L \bar{T}_{q}(1) t=L\left(\frac{b}{a \kappa}\right)^{\nu} \bar{T}_{q}^{1+\nu}(1) t .
\end{gathered}
$$

Recall that

$$
\bar{T}_{q}(1)= \begin{cases}\frac{q^{\nu}\left(\frac{b}{a}\right)^{\frac{\nu}{\mu}}}{a^{\frac{\nu}{\mu}} \frac{\nu}{\nu} c} & \nu>0 \\ \frac{\left(\frac{b}{a}\right)^{-\frac{\nu}{\mu}}-q^{\nu}}{-\frac{\nu}{\mu} c} & \nu<0 \\ \frac{\mu}{c} \ln \left(q\left(\frac{b}{a}\right)^{\frac{1}{\mu}}\right) & \nu=0\end{cases}
$$

then there exists $q>1$ and $\gamma>q^{-1}$ such that

$$
L\left(\frac{b}{a \kappa}\right)^{\nu} \bar{T}_{q}^{1+\nu}(1)+1<\gamma^{-\mu}
$$

provided that

$$
\mu> \begin{cases}\nu+\nu^{2} & \nu>0 \\ 0 & \nu<0 \\ 0 & \nu=0\end{cases}
$$

Then the property $U\left(X\left(t, x_{0}\right)\right)<U\left(x_{0}\right)$ is proven for any $x_{0} \in S_{r}(1)$, and by homogeneity for any $x_{0} \in \mathbb{R}^{n}$ and $t \in\left(0, \bar{T}_{\gamma q}\left(\left\|x_{0}\right\|_{r}\right)\right)$ we have shown that

$$
U\left(X\left(t, x_{0}\right)\right)<U\left(x_{0}\right)
$$

Remark 4. As we can see in the proof above, the analysis of the properties of $U$ is performed on a compact set separated from the origin (between $S_{r}\left(q^{-1}\right)$ and $\left.S_{r}(1)\right)$ and extended globally using the homogeneity arguments. Then the case of $\nu<0$ or even discontinuous dynamics in (1) can be treated similarly if the continuity is lost at the origin only. 


\section{NUMERIC DESIGN}

Let us consider how the Lyapunov function proposed in Theorem 2 can be constructed numerically. By definition, for an $\mathbf{r}$-homogeneous of degree $\mu-\nu$ Lyapunov function $U: \mathbb{R}^{n} \rightarrow \mathbb{R}_{+}$, for any $x \in \mathbb{R}^{n}$ there is a unique $y \in S_{r}(1)$ such that $x=\Lambda_{r}\left(\|x\|_{r}\right) y$ and

$$
U(x)=\|x\|_{r}^{\mu-\nu} U(y) .
$$

Consequently, it is enough to approximate the values of $U$ on $S_{r}(1)$, and next to reconstruct its values globally via homogeneity arguments. This section has two parts: first, pointwise derivation of values of a homogeneous Lyapunov function is discussed; second, an interpolation of its values on $S_{r}(1)$ is presented under mild restrictions leading to a simple numeric procedure.

For a given fixed discretization step $h>0$, let $X_{h}\left(t_{i}, x_{0}\right)$ denote an approximation at instants $t_{i}=i h, i \geq 0$ of the solution $X\left(t, x_{0}\right)$ of (1) using Euler method [6] (any other discretization method can also be used).

\section{A. Pointwise calculation}

For $N>0$, let $\xi_{j} \in S_{r}(1)$ with $j=\overline{1, N}$ form a uniform grid on the unit sphere $S_{r}(1)$, and let us introduce into consideration the following variables:

$$
\begin{gathered}
U_{j}^{h}=h \sum_{i=0}^{J}\left\|X_{h}\left(t_{i}, \xi_{j}\right)\right\|_{r}^{\mu}, \\
J=\tau \underset{i \geq J}{\arg \sup _{i \leq j \leq N}} \sup _{1 \leq j}\left\|X_{h}\left(t_{i}, \xi_{j}\right)\right\|_{r} \leq q^{-1},
\end{gathered}
$$

where $\tau>1$ is a tuning parameter.

Corollary 2. Let Assumption 1 be satisfied. Then there exist $q>1, N>0$ and $h>0$ such that for any $\tau>0$ and $\mu>\max \left\{1, \nu+\nu^{2}\right\}$ :

$$
U_{j}^{h}=U\left(\xi_{j}\right) \quad \forall j=\overline{1, N}
$$

where $U: \mathbb{R}^{n} \rightarrow \mathbb{R}_{+}$is a locally Lipschitz continuous and $\mathbf{r}$-homogeneous of degree $\mu-\nu$ Lyapunov function for the system (1).

Proof: Since all conditions of Theorem 2 are satisfied, then for a sufficiently big $q>1$ a Lyapunov function can be selected in the form given in the theorem:

$$
\widetilde{U}(x)=\int_{0}^{\bar{T}_{q}\left(\left\|x_{0}\right\|_{r}\right)}\|X(t, x)\|_{r}^{\mu} d t .
$$

By the properties of the (explicit) Euler method [6] (see also Theorem 7 in [11] for $\nu>0$ ), for $N$ sufficiently big and $h$ sufficiently small, the following inequalities are satisfied for any $\epsilon>0$ :

$$
\left|U_{j}^{h}-\widetilde{U}\left(\xi_{j}\right)\right| \leq \epsilon \quad \forall j=\overline{1, N} .
$$

By Proposition 2, there exists a family of $\mathbf{r}$-homogeneous of degree $\mu-\nu$ Lyapunov functions $U(x)$ that belong to $\epsilon^{\prime}$-vicinity of $\widetilde{U}(x)$ for some $\epsilon^{\prime}>0$. Since the value of $\epsilon$ can be made arbitrary small by increasing $N$ and decreasing $h$, the result follows.

\section{B. Interpolation}

The result of Corollary 2 shows how pointwise values of a homogeneous Lyapunov function on $S_{r}(1)$ can be derived. Next, based on this result let us make an interpolation. To this end, note that there is always a homogeneous norm such that $S_{r}(1)=\mathbb{S}^{n}=\left\{x \in \mathbb{R}^{n}:\|x\|=1\right\}$ (for example, an implicit definition of a canonical homogeneous norm from [28] can be used), then for the interpolation we will focus on this case only, and denote the geodesic distance on $\mathbb{S}^{n}$ as $g(x, y)=\arccos \left(x^{T} y\right)$ for any $x, y \in \mathbb{S}^{n}$. Following the theory of radial basis function interpolation on the sphere [7], [16], a continuous function $p:[0, \pi] \rightarrow \mathbb{R}$ is (zonal) strictly positive definite on the sphere $\mathbb{S}^{n}$ if for all distinct points $\xi_{j} \in \mathbb{S}^{n}$ with $j=\overline{1, N}$ for all $N>0$, the matrix

$$
\Pi_{N}=\left\{p\left(g\left(\xi_{i}, \xi_{j}\right)\right)\right\}_{i, j=1}^{N}
$$

is positive definite, that is $\lambda_{\min }\left(\Pi_{N}\right)>0$, where $\lambda_{\min }\left(\Pi_{N}\right)$ is the minimum eigenvalue of $\Pi_{N}$ (some examples of such functions can be found in [7], [16], e.g., $p(s)=e^{\cos (s)}$ or $\left.p(s)=(2-\cos (s))^{-1}\right)$. Then, selecting a zonal strictly positive definite function $p$, there exists always $\theta=\left[\theta_{1} \ldots \theta_{N}\right]^{T} \in \mathbb{R}^{N}$ such that

$$
U_{j}^{h}=U\left(\xi_{j}\right) \quad \forall j=\overline{1, N}
$$


for

$$
U(\xi)=\sum_{j=1}^{N} \theta_{j} p\left(g\left(\xi, \xi_{j}\right)\right) .
$$

Indeed, the vector $\theta$ is the solution of the equation

$$
\Upsilon_{N}=\Pi_{N} \theta,
$$

where $\Upsilon_{N}=\left[U_{1}^{h} \ldots U_{N}^{h}\right]^{T} \in \mathbb{R}^{N}$ is the vector composed by the corresponding values of $U_{j}^{h}$ and the matrix $\Pi_{N}$ is nonsingular (symmetric and positive definite) thanks to the properties of the function $p$.

Assumption 2. The function $p\left(g\left(\xi, \xi_{0}\right)\right)$ is Lipschitz continuous with respect to $\xi$ for any $\xi, \xi_{0} \in S_{r}(1)$.

This restriction is satisfied for many examples of zonal strictly positive definite functions $p$ (see [7], [16] and above).

Assumption 3. There exist $\alpha>0$ and $\varrho>0$ such that for any $N>0$

$$
\left\|\Pi_{N}^{-1}\right\|_{2} \leq \frac{\varrho}{N^{1+\alpha}},
$$

where $\|\cdot\|_{2}$ is the induced matrix norm.

Thus, for a sufficiently big value of $N$ (for a sufficiently dense grid on $S_{r}(1)$ ) the derivative of (8) can be made sufficiently small, which implies that the result of Proposition 2 can be applied and the conditions on $\underline{\varepsilon}$ and $\bar{\varepsilon}$ are satisfied:

Theorem 3. Let assumptions 1,2 and 3 be satisfied. Then there exist $q>1, N>0$ and $h>0$ such that for any $\tau>0$ and $\mu>\max \left\{1, \nu+\nu^{2}\right\}$, a locally Lipschitz continuous and $\mathbf{r}$-homogeneous of degree $\mu-\nu$ Lyapunov function for the system (1) can be found on $S_{r}(1)$ in the form (8), where the vector of parameters $\theta$ is the solution of (9).

Proof: Since all conditions of Corollary 2 are satisfied, there exist $q>1, N>0$ and $h>0$ such that for any $\tau>0$ and $\mu>\max \left\{1, \nu+\nu^{2}\right\}$, a locally Lipschitz continuous and $\mathbf{r}$-homogeneous of degree $\mu-\nu$ Lyapunov function $U: \mathbb{R}^{n} \rightarrow \mathbb{R}_{+}$ for the system (1) can be calculated at the points $\xi_{j}$ for all $j=\overline{1, N}$ :

$$
U_{j}^{h}=U\left(\xi_{j}\right) .
$$

From the proof of Theorem 2, the Lipschitz constant of $U$ on the sphere $S_{r}(1)$ is upper bounded by the constant

$$
L_{U}=\bar{T}_{q}(1) L_{S_{r}(1), \bar{T}_{q}(1)},
$$

which depends only on the properties of the system solutions and it is independent on $N$. Due to this property, for any $N>0$ the functions $U$ obtained in Corollary 2 are uniformly upper bounded on $S_{r}(1)$ by some constant $U_{\max } \in \mathbb{R}_{+}$(this bound is related with solutions and not with $N$ ), i.e. $U_{j}^{h} \leq U_{\max }$ for any fixed $q, h, \mu, \tau$ and any $N$. Since $S_{r}(1)$ is a compact set, then the vector $\Upsilon_{N}$ and the matrix $\Pi_{N}$ are elementwise bounded uniformly for any $N>0$. By Assumption 3, the norm of the inverse of $\Pi_{N}$ has an upper bound $\frac{\varrho}{N^{1+\alpha}}$ for some $\varrho>0$, then the solution $\theta$ of (9) is also elementwise upper bounded and decreasing with $N$ :

$$
\begin{aligned}
\|\theta\|_{\max } & \leq\|\theta\| \leq\left\|\Pi_{N}^{-1}\right\|_{2}\left\|\Upsilon_{N}\right\| \\
& \leq N\left\|\Pi_{N}^{-1}\right\|_{2}\left\|\Upsilon_{N}\right\|_{\max } \\
& \leq \varrho N^{-\alpha}\left\|\Upsilon_{N}\right\|_{\max } \leq \varrho N^{-\alpha} U_{\max }
\end{aligned}
$$

where $\|\cdot\|_{\max }$ is the max norm of a vector (elementwise maximum).

Define

$$
\varepsilon(x)=U(x)-\sum_{j=1}^{N} \theta_{j} p\left(g\left(x, \xi_{j}\right)\right)
$$

as the approximation error by (8) of the Lyapunov function $U$. Therefore, due to Assumption 2 there is $\ell>0$ such that

$$
L_{\varepsilon}=\max \left\{L_{U}, \ell \varrho N^{-\alpha} U_{\max }\right\}
$$

is a Lipschitz constant of $\varepsilon$ on $S_{r}(1)$. Since $\varepsilon\left(\xi_{j}\right)=0$ due to (9), then the estimate

$$
\begin{aligned}
\sup _{y \in S_{r}(1)}|\varepsilon(y)| & \leq \sup _{y \in S_{r}(1)}\left|\varepsilon(y)-\varepsilon\left(\xi_{j}\right)\right| \\
& \leq \sup _{y \in S_{r}(1)} L_{\varepsilon}\left|y-\xi_{j}\right|
\end{aligned}
$$


is valid for any $j=\overline{1, N}$. To obtain a tighter upper estimate, for every $y \in S_{r}(1)$, we can consider $j^{*}(y) \in \overline{1, N}$ corresponding to inf $\left|y-\xi_{j}\right|$. So for all $y \in S_{r}(1)$, there is $\kappa>0$ such that

$$
\left|y-\xi_{j^{*}(y)}\right| \leq \frac{\kappa}{N}
$$

since $\xi_{j}, j=\overline{1, N}$ form a uniform grid on the sphere $S_{r}(1)$. Therefore,

$$
\sup _{y \in S_{r}(1)}|\varepsilon(y)| \leq L_{\varepsilon} \frac{\kappa}{N}
$$

is decreasing with $N$. Thus, $\underline{\varepsilon}=\inf _{y \in S_{r}(1)} \varepsilon(y) \geq-\sup _{y \in S_{r}(1)}|\varepsilon(y)| \geq-L_{\varepsilon} \frac{\kappa}{N}$ admits a lower bound strictly increasing in $N$. By definition

$$
\begin{aligned}
\bar{\varepsilon} & =\sup _{y \in S_{r}(1)} D^{+} \varepsilon(y) f(y) \\
& =\sup _{y \in S_{r}(1)} D^{+}\left(U(y)-\sum_{j=1}^{N} \theta_{j} p\left(g\left(y, \xi_{j}\right)\right)\right) f(y) \\
& \leq \sup _{y \in S_{r}(1)}\left|D^{+} \sum_{j=1}^{N} \theta_{j} p\left(g\left(y, \xi_{j}\right)\right) f(y)\right|
\end{aligned}
$$

since $D^{+} U(y) f(y)<0$ for all $y \in S_{r}(1)$. As it is shown in Proposition 1.1.a of [9]:

$$
\sup _{y \in S_{r}(1)}\left|D^{+} \sum_{j=1}^{N} \theta_{j} p\left(g\left(y, \xi_{j}\right)\right) f(y)\right| \leq \ell\|\theta\|_{\max } \sup _{y \in S_{r}(1)}\|f(y)\|,
$$

and

$$
\bar{\varepsilon} \leq \ell \varrho N^{-\alpha} U_{\max } \sup _{y \in S_{r}(1)}\|f(y)\|
$$

is a strictly decreasing function of $N$. Therefore, the values $-\underline{\varepsilon}$ and $\bar{\varepsilon}$ are decreasing with $N$ for the approximation (8), and there is a sufficiently big $N>0$ such that the conditions of Proposition 2 are satisfied.

Note that due to the introduced assumptions and to properties of the function $U$ in (8), the directional derivative $D^{+} U(y) f(y)$ is well defined for all $y \in S_{r}(1)$. Thanks to the $\mathbf{r}$-homogeneity of $f$ with degree $\nu$ and to the $\mathbf{r}$-homogeneity of $U$ of degree $\mu-\nu$,

$$
D^{+} U(x) f(x)=\|x\|_{r}^{\mu} D^{+} U(y) f(y)
$$

for any $x \in \mathbb{R}^{n}$, where $y \in S_{r}(1)$ is such that $x=\Lambda_{r}\left(\|x\|_{r}\right) y$, and $D^{+} U(x) f(x)$ is well-defined for all $x \in \mathbb{R}^{n}$.

\section{EXAMPLES}

For simplicity of presentation, the case $n=2$ is investigated in this section, and the following expressions and values of parameters are used for computation of a homogeneous Lyapunov function:

$$
\begin{gathered}
\mu=\max \left\{1, \nu+\nu^{2}\right\}+0.15, \\
q=2, \tau=1.25, h=0.01, \\
p(s)=|s| .
\end{gathered}
$$

In the examples below, these values have been fixed and the value of $N$ was increased (similarly $h^{-1}$ and $q$ may be gradually augmented) until convergence of the procedure.

\section{A. Nonlinear system 1}

Consider (1) with

$$
f(x)=\left[\begin{array}{c}
x_{2}-\left\lceil x_{1}\right\rfloor^{5} \\
-\left\lceil x_{1}\right\rfloor^{1 \frac{2}{3}}
\end{array}\right]
$$

for $x \in \mathbb{R}^{2}$, then $\nu=\frac{2}{3}$ for $\mathbf{r}=\left[\frac{1}{3} 1\right]$. The results of simulation are shown in Fig. 1 for $N=50$. The state trajectories are presented in Fig. 1,a. The obtained values of $U$ are plotted in Fig. 1,b (the bold points represent $U_{j}^{h}$ ), the derivative of $U$ is given in Fig. 1,c. As we can conclude from these results the obtained values correspond to a Lyapunov function, and it is homogeneous by construction. 

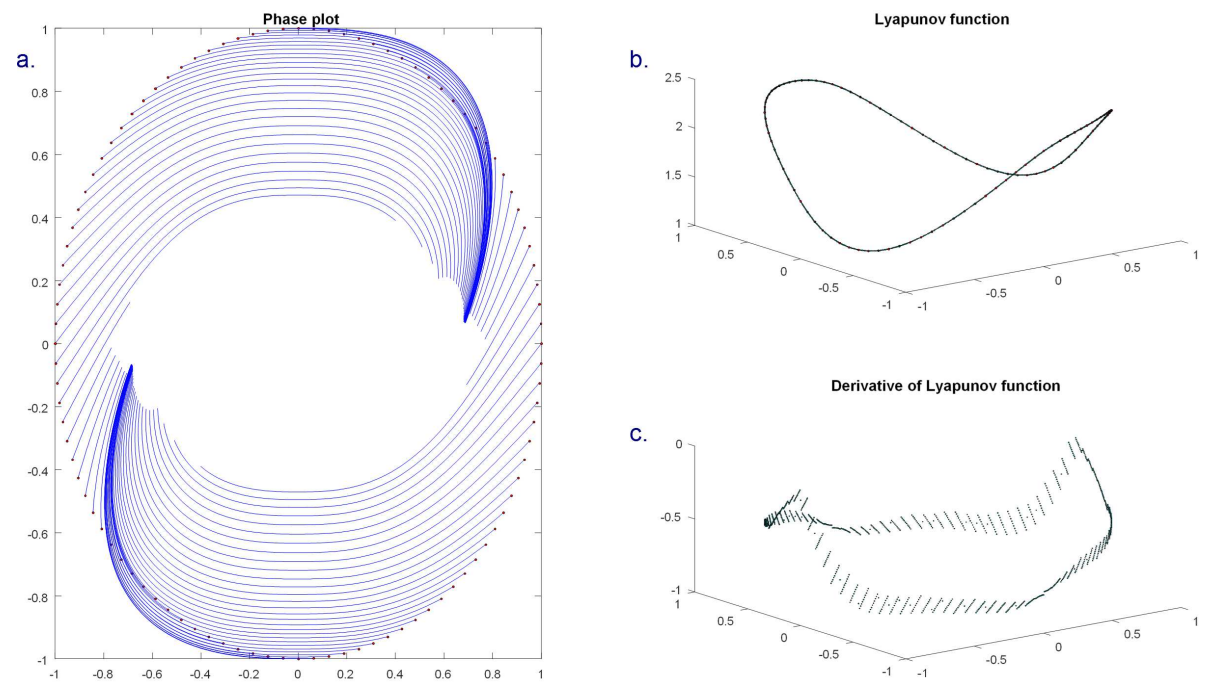

Figure 1. Results of calculations for nonlinear system 1: a. State trajectories; b. The level of Lyapunov function $U(y)$ for $y \in S_{r}(1)$; c. The values of the derivative $D^{+} U(y) f(y)$ for $y \in S_{r}(1)$
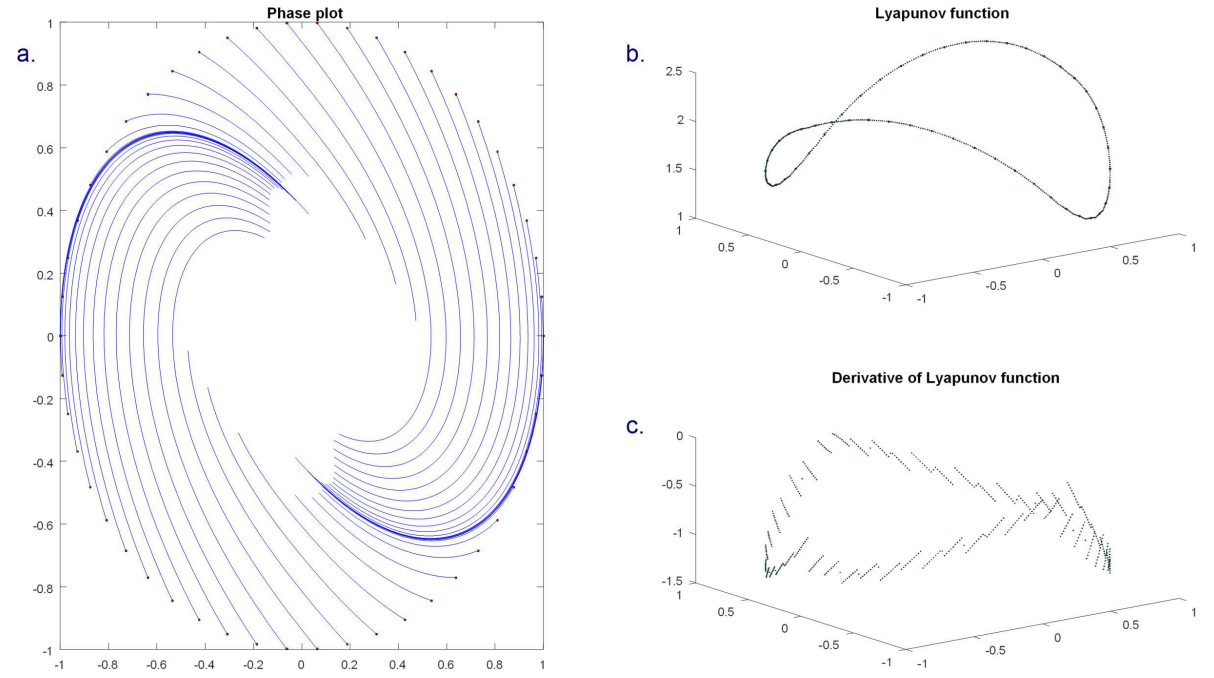

Figure 2. Results of calculations for nonlinear system 2: a. State trajectories; b. The level of Lyapunov function $U(y)$ for $y \in S_{r}(1)$; c. The values of the derivative $D^{+} U(y) f(y)$ for $y \in S_{r}(1)$

\section{B. Nonlinear system 2}

Consider (1) with

$$
f(x)=\left[\begin{array}{c}
x_{2} \\
-\left\lceil x_{1}\right\rfloor^{3}-\left\lceil x_{2}\right\rfloor^{1.5}
\end{array}\right]
$$

for $x \in \mathbb{R}^{2}$, then $\nu=0.5$ for $\mathbf{r}=[0.51]$. The results of simulation are shown in Fig. 2 for $N=100$ (trajectories in Fig. 2,a, the values of $U$ in Fig. 2,b and the derivative in Fig. 2,c). The results of these calculations also confirm our theoretical findings.

\section{CONCLUSION}

The problem of construction of a homogeneous Lyapunov function for an asymptotically stable homogeneous system is revisited. First, for systems with nonnegative degree of homogeneity, several expressions of homogeneous Lyapunov functions are proposed, which depend explicitly on the system solutions (on finite or infinite intervals of time). Second, a procedure is presented, which under some technical assumptions ensures that a homogeneous Lyapunov function can be numerically constructed. The results are illustrated by simulations for linear and nonlinear cases. The proposed numeric design of Lyapunov functions can be used for verification of stability of homogeneous systems. Future directions of research 
include extensions of our approach for the cases of negative degree and discontinuous dynamics. Guidelines for parameter tuning have also to be developed.

\section{REFERENCES}

[1] A. Bacciotti and L. Rosier. Liapunov Functions and Stability in Control Theory, volume 267 of Lecture Notes in Control and Inform. Sci. Springer, Berlin, 2001.

[2] E. Bernuau, D. Efimov, W. Perruquetti, and A. Polyakov. On homogeneity and its application in sliding mode control. Journal of the Franklin Institute, 351(4):1866-1901, 2014.

[3] E. Bernuau, A. Polyakov, D. Efimov, and W. Perruquetti. Verification of ISS, iISS and IOSS properties applying weighted homogeneity. Systems \& Control Letters, 62(12):1159-1167, 2013.

[4] N. P. Bhatia and G. P. Szegö. Dynamical Systems: Stability Theory and Applications. Springer-Verlag, Berlin, 1967.

[5] C. Briat. Linear Parameter-Varying and Time-Delay Systems. Advances in Delays and Dynamics. Springer-Verlag, Berlin, 2015.

[6] J. C. Butcher. Numerical Methods for Ordinary Differential Equations. John Wiley \& Sons, New York, 2003.

[7] E. W. Cheney. Approximation and Interpolation on Spheres, pages 47-53. Springer Netherlands, Dordrecht, 1995.

[8] N. G. Chetaev. The Stability of Motion. Pergamon Press, New York, 1961. (English translation).

[9] F. H. Clarke, Y. S. Ledyaev, R. J. Stern, and P. R. Wolenski. Nonsmooth Analysis and Control Theory, volume 178 of Graduate Texts in Mathematics. Springer-Verlag, New York, 1998.

[10] D. Efimov and W. Perruquetti. Conditions of oscillations and multi-homogeneity. Mathematics of Control, Signals, and Systems, 28(3):1-37, 2016.

[11] D. Efimov, A. Polyakov, A. Levant, and W. Perruquetti. Realization and discretization of asymptotically stable homogeneous systems. IEEE Transactions on Automatic Control, 62(11):5962-5969, 2017.

[12] D. Efimov, R. Ushirobira, J.A. Moreno, and W. Perruquetti. On the numerical construction of homogeneous lyapunov functions. In Proc. 56th IEEE Conference on Decision and Control (CDC), Melbourne, 2017.

[13] A.L. Fradkov and A.Yu. Pogromsky. Introduction to oscillations and chaos. World Scientific, Singapore, 1998.

[14] P. Giesl and S. Hafstein. Review on computational methods for Lyapunov functions. Discrete and Continuous Dynamical Systems - Series B, 20(8):2291-2331, 2015.

[15] W. Hahn. Stability of Motion. Springer, Berlin, 1967.

[16] S. Hubbert. Radial basis function interpolation on the sphere. PhD thesis, Imperial College London, 2002.

[17] A. Isidori. Nonlinear control systems: An Introduction. Springer-Verlag, Berlin, 2nd edition, 1989.

[18] H. K. Khalil. Nonlinear Systems. Prentice Hall, Upper Saddle River, New Jersey, 3rd edition, 2002.

[19] N.N. Krasovskii. Stability of Motion: Applications of Lyapunov's Second Method to Differential Systems and Equations With Delay. Stanford University Press, 1963.

[20] J. Kurzweil. On the reversibility of the first theorem of Lyapunov concerning the stability of motion. Czechoslovak Mathematical Journal, 5:382-398, 1955.

[21] Y. Lin, E. D. Sontag, and Y. Wang. A smooth converse Lyapunov theorem for robust stability. SIAM J. Control Optimization, 34:124-160, 1996.

[22] A.M. Lyapunov. The general problem of the stability of motion. Taylor \& Francis, London, 1992. Translated by A. T. Fuller.

[23] J. L. Massera. On Liapounoff's conditions of stability. Annals of Mathematics, 50:705-721, 1949.

[24] H. Nakamura, Y. Yamashita, and H. Nishitani. Smooth lyapunov functions for homogeneous differential inclusions. In Proc. 41st SICE Annual Conference, volume 3, pages 1974-1979. IEEE, 2002.

[25] M. M. Peet and A. Papachristodoulou. A converse sum of squares lyapunov result with a degree bound. IEEE Transactions on Automatic Control, 57(9):2281-2293, 2012.

[26] K. P. Persidskii. On a theorem of Liapunov. C. R. (Dokl.) Acad. Sci. URSS, 14:541-543, 1937.

[27] A. Polyakov. Nonlinear feedback design for fixed-time stabilization of linear control systems. IEEE Transactions on Automatic Control, 57(8):2106$2110,2012$.

[28] A. Polyakov, J.-M. Coron, and L. Rosier. On finite-time stabilization of evolution equations: A homogeneous approach. In Proc. IEEE CDC, pages 3143-3148. IEEE, 2016.

[29] L. Rosier. Homogeneous lyapunov function for homogeneous continuous vector field. Systems \&Control Lett., 19:467-473, 1992.

[30] R. Sepulchre, M. Jankovic, and P. V. Kokotović. Constructive nonlinear control. Springer-Verlag, NY, 1997.

[31] E.D. Sontag. A "universal" construction of Arstein's theorem on nonlinear stabilization. Systems \& Control Letters, 12:542-550, 1989.

[32] T. Yoshizawa. On the stability of solutions of a system of differential equations. Memoirs of the College of Science, University of Kyoto, Series A: Mathematics, 29:27-33, 1955.

[33] V. I. Zubov. Methods of A.M. Lyapunov and Their Application. Stechert-Hafner Service Agency, 1964. 